\title{
Differential Polynomial Identities of Upper Triangular Matrices Under the Action of the Two-Dimensional Metabelian Lie Algebra
}

\author{
Onofrio M. Di Vincenzo ${ }^{1} \cdot$ Vincenzo Nardozza ${ }^{2}$
}

Received: 5 August 2019 / Accepted: 24 November 2020 / Published online: 23 January 2021

(C) The Author(s) 2021

\begin{abstract}
We study the differential polynomial identities of the algebra $U T_{m}(F)$ under the derivation action of the two dimensional metabelian Lie algebra, obtaining a generating set of the $T_{L^{-}}$ ideal they constitute. Then we determine the $S_{n}$-structure of their proper multilinear spaces and, for the minimal cases $m=2,3$, their exact differential codimension sequence.
\end{abstract}

Keywords Differential polynomial identity · Upper triangular matrices · Differential codimensions $\cdot$ Lie algebras · Derivation action

Mathematics Subject Classification (2010) Primary 16R50 - Secondary 16W25 · 17B60

\section{Introduction}

Differential polynomial identities constitute a natural and direct generalization of the notion of polynomial identities of an algebra. They take into account the identical relations holding for an algebra whose structure is enriched by the action of a Lie algebra of derivations, and constitute a trending topic within the PI-Theory of associative algebras (see [3] and its bibliography). Besides ordinary and differential polynomial identities, other similar notions have been brought up: graded identities, trace identities, $*$-identities, $H$-identities all have their own relevance, not just for their own intrinsic interest. In fact they are generally easier

Presented by: Michel Van den Bergh

Partially supported by GNSAGA 2019

Vincenzo Nardozza

vincenzo.nardozza@uniba.it

Onofrio M. Di Vincenzo

onofrio.divincenzo@unibas.it

1 Dipartimento di Matematica, Informatica ed Economia, Università degli Studi della Basilicata, viale dell'Ateneo Lucano 10, 85100 Potenza, Italia

2 Dipartimento di Matematica, Università degli Studi di Bari, via Orabona 4, 70125 Bari, Italia 
to discover, and each of these types of identities completely determines the ordinary ones, at least in principle.

It must be said that in present days an effort towards a unifying approach among different types of identities is being pursued. It took its steps from Berele's influential paper [4], and has its homeland in the settings of Hopf-algebra actions. For instance, in the specific case of differential identities of an algebra $A$ under the derivations of a Lie algebra $L$, the Hopf algebra involved is the universal enveloping algebra of $L$. Even when no straight Hopf-algebra action is possible, such as for instance for $*$-identities, one can consider a generalized Hopf-algebra action to keep on track ([4], Remark at page 878. See also [2], Section 7). Of course this does not mean that differences cease to exist: as always happens, general theories are built upon concrete situations, so differential identities, as well as other types of identities, still maintain their characteristic features when dealing with specific problems.

In the present paper we consider the smallest nonabelian Lie algebra, namely the metabelian two-dimensional Lie algebra $L$, acting on the algebra $U T_{m}(F)$ by derivations. We give a faithful representation of $L$ as the Lie algebra generated by two suitable inner derivations $\delta, \epsilon$ of $U T_{m}(F)$, which turns out to be very convenient to computational tasks. Our main result consists in providing a full list (Definition 3.4) of differential polynomials, generating all differential polynomial identities of $U T_{m}(F)$ with respect to this $L$-action (Theorem 4.11). Then we pass to study the differential multilinear spaces related to $U T_{m}(F)$ through their $S_{n}$-structure. The key to these results is afforded by the use of proper polynomials, a powerful tool re-discovered and developed mainly by Drensky, who pointed out their extreme usefulness in several papers. More precisely, we determine the proper differential cocharacter sequence of $U T_{m}(F)$ recursively (Proposition 5.1 and Theorem 5.8) and explicitly (Corollary 5.9).

The last section of our paper is devoted to work out the small cases $m=2$ and $m=3$. In the former we regain the results of [11] as byproduct of our general description. On the other hand, $U T_{2}(F)$ is too small to depict the effective impact of the $L$-action on the differential polynomial identities in general cases. All its characteristic features are instead already present when $m=3$, so this case is worth studying to get a more concrete idea of the general one.

\section{Algebras with Derivations and Differential Polynomial Identities}

Throughout the paper let $F$ denote a field. With the term $F$-algebra we mean an associative $F$-algebra. If $A$ is an $F$-algebra, let $\operatorname{End}_{F}(A)$ denote the full algebra of $F$-linear trasformations from $A$ to itself. A derivation on $A$ is any $d \in \operatorname{End}_{F}(A)$ satisfying $\left(a_{1} \cdot a_{2}\right)^{d}=a_{1}^{d} \cdot a_{2}+a_{1} \cdot a_{2}^{d}$ for all $a_{1}, a_{2} \in A$. We will adopt the exponential notation for derivations throughout the paper. The set of all derivations on $A$ is a vector subspace of $\operatorname{End}_{F}(A)$, turned into a Lie algebra with respect the usual Lie product $[f, g]:=f g-g f$ between maps, and is denoted $\operatorname{Der}(A)$. Notice that, since we use a right action, linear maps are composed from left to right. If $L$ is a Lie algebra over $F$, it acts on A as an algebra of derivations if $A$ is a Lie module of $L$. Actually, denoting $\mathfrak{L}$ the universal enveloping algebra $U(L)$ of $L$, this amounts to turn $A$ into a right $\mathfrak{L}$-module. We briefly recall that $\mathfrak{L}$ is uniquely determined, and the Poincarè-Birkhoff-Witt Theorem provides a natural embedding of $L$ in $\mathfrak{L}$ and an explicit basis of $\mathfrak{L}$ starting from any linearly ordered basis $\mathscr{B}$ of $L$, namely the set $\mathfrak{B}$ of all semistandard products $b_{1} b_{2} \ldots b_{k}$ (that is: $b_{1} \leqslant b_{2} \leqslant \cdots \leqslant b_{k}$ ) of elements $b_{i} \in \mathscr{B}$. 
For a fixed Lie algebra $L$, it is possible to define a free object for the class of $F$-algebras on which $L$ acts as an algebra of derivations ( $L$-algebras, for short). Let us start with a countable set of free indeterminates $X$, and consider the tensor algebra of the vector space $F X \otimes_{F} \mathfrak{L}$. It is a free algebra generated by the simple tensors $x \otimes p$ for $x \in X$ and $p \in \mathfrak{B}$. We will denote $x \otimes p$ by $x^{p}$ and call it a letter; moreover, we may identify the indeterminate $x \in X$ with the letter $x \otimes 1$. We will denote by $X^{L}$ the set of free generators (letters) $x^{p}$, and by $F\left\langle X^{L}\right\rangle$ the free algebra they generate. Since $X \subseteq X^{L}$, the free algebra $F\langle X\rangle$ is a subalgebra of $F\left\langle X^{L}\right\rangle$. Actually, $F\left\langle X^{L}\right\rangle$ is free on the set $X$ in the class of $L$-algebras, in the sense that if $A$ is any $L$-algebra, any map $v: X \rightarrow A$ can be uniquely extended to an $L$ algebra homomorphism $\bar{v}: F\left\langle X^{L}\right\rangle \rightarrow A$, that is an algebra homomorphism extending $v$ and commuting with the derivation action of $L$ (and $\mathfrak{L}$ ). Thus $F\left\langle X^{L}\right\rangle$ is called the free L-algebra on $X$. Each element of $F\left\langle X^{L}\right\rangle$ can be thought as a polynomial in the noncommutative letters $x^{p}$. If $p \neq 1$ we will say $x^{p}$ is a differential letter, while $x$ is an ordinary letter. When $X=\left\{x_{i}: 1 \leqslant i \in \mathbb{N}\right\}$, we say that the $i$ is the name of the letter $x_{i}^{p}$. A polynomial $f \in F\left\langle X^{L}\right\rangle$ is called ordinary if just ordinary letters occur in $f$, otherwise it is called differential.

Now let $A$ be an $L$-algebra. The following notions have been outlined in [4] in the general settings of Hopf algebra actions, and employed in [11] in the more specific situation of a derivation action. Let $T_{L}(A)$ be the set constituted by the differential polynomials laying in the kernel of all $L$-homomorphisms $\varphi: F\left\langle X^{L}\right\rangle \rightarrow A$. Then $T_{L}(A)$ is an ideal of $F\left\langle X^{L}\right\rangle$ invariant under all the $L$-endomorphisms of $F\left\langle X^{L}\right\rangle$, called the $T_{L}$-ideal of $A$, and its elements are called the $L$-polynomial identities of $A$. Notice that the ordinary $T$-ideal of $A$, namely the set $T(A)$ of all (ordinary) polynomials of $F\langle X\rangle$ vanishing under all algebra homomorphisms from $F\langle X\rangle$ to $A$, is contained in $T_{L}(A)$. If $\mathscr{G}$ is any subset of $F\left\langle X^{L}\right\rangle$, the least $T_{L}$-ideal of $F\left\langle X^{L}\right\rangle$ containing $\mathscr{G}$ is called the $T_{L}$-ideal generated by $\mathscr{G}$. One of the main tasks in studying the differential polynomial identities of an algebra $A$ is to find a generating set for $T_{L}(A)$.

In order to describe $T_{L}(A)$ a smaller set of differential polynomials is needed when char. $F=0$. In fact standard Vandermonde arguments and multilinearization process allow reducing the description to listing the multilinear differential polynomials $T_{L}(A)$ contains. Recall that a polynomial $f \in F\left\langle X^{L}\right\rangle$ is multilinear of degree $n$ if each name among $1, \ldots, n$ occurs exactly once in any monomial of $f$, and no other name occurs. As in [11] we denote $P_{n}^{L}:=\operatorname{span}_{F}\left\langle x_{\sigma(1)}^{p_{1}} \ldots x_{\sigma(n)}^{p_{n}} \mid \sigma \in S_{n}, p_{i} \in \mathfrak{B}\right\rangle$, the space of multilinear differential polynomials of degree $n$. Moreover, let $P^{L}:=\bigcup_{n \geqslant 0} P_{n}^{L}$. Then $T_{L}(A)$ is the least $T_{L}$-ideal of $F\left\langle X^{L}\right\rangle$ containing the set $P^{L} \cap T_{L}(A)$. In other words, $T_{L}(A)$ is generated, as $T_{L}$-ideal, by the multilinear $L$-polynomials it contains. Actually, each $P_{n}^{L}$ is more than a vector space: the natural left $S_{n}$-action $\sigma\left(x_{i}^{p}\right):=x_{\sigma(i)}^{p}$ for all $\sigma \in S_{n}$ turns $P_{n}^{L}$ into an $S_{n}$-module, having $P_{n}^{L} \cap T_{L}(A)$ as a submodule. The factor module $P_{n}^{L}(A):=P_{n}^{L} /\left(P_{n}^{L} \cap T_{L}(A)\right)$ is therefore an $S_{n}$-module as well, and its module structure can be described through its $S_{n}$-character, called the $n$-th $L$-cocharacter of $T_{L}(A)$. Though it essentially describes the structure of the non identity multilinear polynomials, the intere $S_{n}$-structure of $P_{n}^{L} \cap T_{L}(A)$ can be recovered by complete reducibility. The associated number sequence $c_{n}^{L}(A):=\operatorname{dim} P_{n}^{L}(A)$, for $n \in \mathbb{N}$, is called the $L$-codimension sequence of $A$.

When dealing with unitary algebras, a further strong reduction is actually available. For any $z_{1}, z_{2} \in X^{L}$ define the commutators of length 2 by $\left[z_{1}, z_{2}\right]:=z_{1} z_{2}-z_{2} z_{1}$ and, for $k \geqslant 3$, recursively define the commutators of length $k$ by $\left[z_{1}, z_{2}, \ldots, z_{k}\right]:=$ $\left[\left[z_{1}, z_{2}, \ldots, z_{k-1}\right], z_{k}\right]$. The unitary subalgebra of $F\left\langle X^{L}\right\rangle$ generated by the commutators of any length will be denoted $B^{L}$, and we will call its elements $L$-proper polynomials. The 
relation between $F\left\langle X^{L}\right\rangle$ and $B^{L}$ can be clearly described through Lie algebras: let $\mathscr{L}$ be the free Lie algebra generated by $X^{L}$ and let $\mathscr{L}^{\prime}=[\mathscr{L}, \mathscr{L}]$ be its derived ideal. Then $\mathscr{L}^{\prime}$ is also a free Lie algebra, and $\mathscr{L}$ is spanned by $X^{L}$ modulo $\mathscr{L}^{\prime}$. By Witt's Theorem, $F\left\langle X^{L}\right\rangle$ is the universal enveloping algebra of $\mathscr{L}$, while $B^{L}$ is the universal enveloping algebra of $\mathscr{L}^{\prime}$. Furthermore, let us fix a linear order in $X^{L}$ such that the ordinary letters precede the differential ones. Then the semistandard commutators $\left[z_{1}, z_{2}, \ldots, z_{k}\right]$ (that is such that $z_{1}>z_{2} \leqslant z_{3} \leqslant \cdots \leqslant z_{k}$ ) form a basis for $\mathscr{L}^{\prime}$ (see [1], Corollary of Proposition 8, (ii), p. $55)$, and can be completed to a basis for $\mathscr{L}$ by adding the elements of $X^{L}$. The linear ordering on $X^{L}$ can be extended to a total order on this basis such that elements of $X^{L}$ precede any commutator. Then the semistandard polynomials $w b$, where $w$ is a semistandard monomial on $X^{L}$ and $b$ is a semistandard sequence of the $\mathscr{L}^{\prime}$-basis, constitute a basis for $F\left\langle X^{L}\right\rangle$ by the Poincarè-Birkhoff-Witt Theorem.

We actually need just polynomials which are proper with respect to ordinary letters, that is elements of the subalgebra $B_{X}^{L}$ of $F\left\langle X^{L}\right\rangle$ generated by commutators and differential letters. Thus we may consider the differential letters as commutators of length 1 , similarly to what has been made in [6], and consider normal semistandard commutators (nssc's for short) of length $\geqslant 1$ : a commutator $\left[z_{1}, z_{2}, \ldots, z_{k}\right]$ is normal if at most a single differential letter occurs in it, and in this case it is $z_{1}$. It has been proved in [6], Proposition 7, that the nssc's constitute an $F$-basis for $B_{X}^{L}$. It can be proved that $T_{L}(A)$ is generated by the $X$ proper polynomials it contains. Even simpler, we just need multilinear proper polynomials. In fact, let $\Gamma_{n}^{L}:=P_{n}^{L} \cap B_{X}^{L}$ for all $n \in \mathbb{N}$ and let $\Gamma$ be the union of these sets. Then $T_{L}(A)$ is generated by $\Gamma \cap T_{L}(A)$.

Although proper polynomials could be dealt with in much greater generality (see [7], section 4.3 for the basic definitions and results on proper polynomials; polynomials proper with respect to a distinct set of letters were introduced in [8], Section 2), in this paper we shall deal with multilinear polynomials only. As a consequence, the nssc's we are going to deal with are actually normal standard commutators (nsc for short), that is nssc's such that $z_{1}>z_{2}<\cdots<z_{n}$. Both in order to keep the paper as self-contained as possible and to give a proof within our restricted assumptions, we prove

Lemma 2.1 $T_{L}(A)$ is generated as $T_{L}$-ideal by $\Gamma \cap T_{L}(A)$.

Proof Let $I$ be the $T_{L}$-ideal generated by $\Gamma \cap T_{L}(A)$. Then $I \subseteq T_{L}(A)$. Since $P \cap T_{L}(A)$ generates $T_{L}(A)$, if $I \neq T_{L}(A)$ then there must be polynomials $f \in P_{n}$ such that $f \in T_{L}(A)$ but $f \notin I$, and we may choose one of minimal degree $n$. It has to be a linear combination of products $w g_{w}$ where $w$ is a standard monomial on a subset of the ordinary letters $x_{1}, \ldots, x_{n}$ and $g_{w}$ is a product of commutators in the remaining letters, say

$$
f=\sum_{w} \alpha_{w} x_{1} w g_{w}+\sum_{u} \alpha_{u} u g_{u}
$$

where none among the monomials $w, u$ involves the letter $x_{1}$. Now let us consider the $L$ endomorphism $\varphi$ of $F\left\langle X^{L}\right\rangle$ sending $x_{1}$ in $1_{F}$ and fixing all other indeterminates. Then $\varphi(f) \in T_{L}(A)$. By the way, just the polynomials $w g_{w}$ may contribute to $f\left(1, x_{2} \ldots, x_{n}\right)$ : indeed $x_{1}$ participates in each $g_{u}$, either occurring in a commutator of length $\geqslant 2$ or as a 1-commutator, that is a single derived letter. In both cases, $g_{u}$ evaluates to zero. Therefore $\varphi(f)=\sum_{w} \alpha_{w} w g_{w}$ is still multilinear in the remaining letters, belongs to $T_{L}(A)$ and has degree $n-1$. Renaming the letters, we get an element in $P_{n-1} \cap T_{L}(A)$. By minimality, it must be in $I$. Therefore $\varphi(f) \in I$, hence $\sum_{w} x_{1} w g_{w} \in I$, so $f \equiv \sum_{u} \alpha_{u} u g_{u}(\bmod I)$. 
Since we may repeat the process on the minimal letter occurring among the $u^{\prime} s$, after finite steps we get $f \equiv 0(\bmod I)$, a contradiction.

\section{A Spanning Set for $\Gamma_{n}^{L}\left(U_{m}\right)$}

In this paper we are interested in the only nonabelian 2-dimensional Lie algebra over $F$. It is also called the metabelian Lie algebra of dimension two, although this term may be confusing. Indeed while in most cases the word metabelian means 2-solvable (see [1], p. 27, and [7], Remark 2.1.17, (iii) p. 22, but also, p. 117 for metabelian groups), sometimes it is intended as 2-nilpotent (see [10], Definition 1.1).

It is well known (see for instance [12], p. 11) that if $L$ is a two-dimentional noncommutative Lie algebra then there exists a basis $\{a, b\}$ of $L$ such that $[a, b]=a$. Any linear map $\theta: L \rightarrow \operatorname{Der}\left(U T_{m}(F)\right)$ such that $[\theta(a), \theta(b)]=\theta(a)$ is a Lie-homomorphism, and induces a derivation action of $L$ on $U T_{m}(F)$. Notice also that $\theta$ is injective as soon as $\theta(a) \neq 0$. In this case we denote $U_{m}$ the algebra $U T_{m}(F)$ under the $L$-action, to distinguish this structure from the natural algebra structure, which we will continue to denote by $U T_{m}(F)$. By [5], all derivations of $U T_{m}(F)$ are inner, so $\theta$ can be defined as soon as a couple of elements $x, y \in U T_{m}(F)$ such that $\left[D_{x}, D_{y}\right]=D_{x}$ is chosen, where $D_{v}:=[\cdot, v]$ is the inner derivation defined by $v \in U T_{m}(F)$. This amounts to turn $U T_{m}(F)$ into a right $\mathfrak{L}$-module. Our real concern is, in fact, in the $\mathfrak{L}$-action on $U_{m}$, in order to determine the differential identities of $U_{m}$.

From now on, we denote by $\delta, \epsilon$ the inner derivations of $U T_{m}(F)$ induced by $e_{1 m}$ and $e_{m m}$ respectively, that is $u^{\delta}:=\left[u, e_{1 m}\right]$ and $u^{\epsilon}=\left[u, e_{m m}\right]$ for all $u \in U T_{m}(F)$. Then $\delta$ and $\epsilon$ satisfy the relations

$$
\epsilon^{2}=\epsilon, \quad \delta^{2}=0, \quad \delta \epsilon=\delta, \quad \epsilon \delta=0,
$$

hence $[\delta, \epsilon]=\delta$. Therefore $\delta$ and $\epsilon$ generate a Lie subalgebra of the (Lie) algebra of $F$ endomorphisms of $U T_{m}(F)$ isomorphic to $L$.

While the relation $[\delta, \epsilon]=\delta$ suffices to establish a Lie isomorphism between $L$ and the Lie subalgebra of $\operatorname{End}_{F}\left(U T_{m}(F)\right)$ generated by the nonzero derivations $\delta$ and $\epsilon$, the other relations, namely $\epsilon^{2}=\epsilon, \delta^{2}=0$ and $\delta \epsilon=\delta$ (which, together with $[\delta, \epsilon]=\delta$, implies $\epsilon \delta=$ 0 ) affect the concrete action of $\mathfrak{L}$ over $U_{m}$ : it turns out that the relations $a^{2}, b^{2}-b, a b-b, b a$ generate the kernel $\mathfrak{K}$ of this action. Therefore the $\mathfrak{L}$-module structure of $U_{m}$ is equivalent to the (right) module structure afforded by the factor algebra $\mathfrak{L} / \mathfrak{K}$ spanned by $1_{F}, \delta$ and $\epsilon$.

Example 3.1 Assign $\delta:=\left[\cdot, e_{23}\right]$ and $\epsilon:=\left[\cdot, e_{33}\right]$. Again $\theta: L \rightarrow \operatorname{Der}\left(U T_{m}(F)\right)$, defined by $\theta(a)=\delta$ and $\theta(b)=\epsilon$, is an injective Lie homomorphism, since $[\delta, \epsilon]=\delta$. By the way, while still holding $\delta^{2}=0$, it is no longer true that $\epsilon^{2}=\epsilon, \epsilon \delta=0$ and $\delta \epsilon=\delta$ hold. These facts alter the computational behaviour of the $\mathfrak{L}$-action on $U_{m}$ because the generators of the kernel change. The resulting $\mathfrak{L}$-module is equivalent to the previous one, that is the factor algebra is isomorphic to the previous one, but the computations are more complex.

As a consequence of our choice, we get the following differential identities of $U_{m}$ :

$$
x^{\epsilon^{2}}-x^{\epsilon}, \quad x^{\delta^{2}}, \quad x^{\delta \epsilon}-x^{\delta}, \quad x^{\epsilon \delta} .
$$

Therefore the only nontrivial differential indeterminates involved in $F\left\langle X^{L}\right\rangle$ modulo $T_{L}\left(U_{m}\right)$ are just $x^{\epsilon}$ and $x^{\delta}$. It holds 
Lemma 3.2 The polynomials $x_{1}^{\epsilon} x_{2}^{\epsilon}, x_{1}^{\delta} x_{2}^{\delta}, x_{1}^{\epsilon} x_{2}^{\delta}, x_{1}^{\delta} x_{2}^{\epsilon}$ are in the $T_{L}$-ideal generated by $x^{\epsilon^{2}}-x^{\epsilon}, x^{\delta^{2}}, x^{\delta \epsilon}-x^{\delta}$ and $x^{\epsilon \delta}$.

Proof Evaluating $\left(x_{1} x_{2}\right)^{\epsilon \delta}$ one has

$$
0 \equiv\left(x_{1} x_{2}\right)^{\epsilon \delta}=\left(x_{1}^{\epsilon} x_{2}+x_{1} x_{2}^{\epsilon}\right)^{\delta}=x_{1}^{\epsilon \delta} x_{2}+x_{1}^{\epsilon} x_{2}^{\delta}+x_{1}^{\delta} x_{2}^{\epsilon}+x_{1} x_{2}^{\epsilon \delta} \equiv x_{1}^{\epsilon} x_{2}^{\delta}+x_{1}^{\delta} x_{2}^{\epsilon}
$$

modulo the listed identities. Replacing $x_{1}$ by $x_{1}^{\epsilon}$ one has $0 \equiv x_{1}^{\epsilon^{2}} x_{2}^{\delta}+x_{1}^{\epsilon \delta} x_{2}^{\epsilon} \equiv x_{1}^{\epsilon} x_{2}^{\delta}$, hence $x_{1}^{\delta} x_{2}^{\epsilon} \equiv 0$ as well.

Then just evaluate the polynomials $\left(x_{1} x_{2}\right)^{\epsilon^{2}}$ and $\left(x_{1} x_{2}\right)^{\delta^{2}}$ to get the remaining monomial identities.

There are further basic $L$-identities of $U_{m}$, which can be easily checked:

- $x^{\delta}\left[x_{1}, x_{2}\right],\left[x_{1}, x_{2}\right] x^{\delta}$;

- $\left[x_{1}, x_{2}\right]^{\delta}$. A different writing of this polynomial is $\left[x_{1}^{\delta}, x_{2}\right]+\left[x_{1}, x_{2}^{\delta}\right]$;

- $x^{\epsilon}\left[x_{1}, x_{2}\right]$

- $\left[x_{1}, y_{1}\right] \ldots\left[x_{m}, y_{m}\right]$;

- $\left[x_{1}, y_{1}\right] \ldots\left[x_{m-1}, y_{m-1}\right] x^{\epsilon}$.

The last basic $L$-identity of $U_{m}$, involving a product of $m-1$ commutators, is the following:

Lemma $3.3\left[x_{1}, y_{1}\right] \ldots\left[x_{m-2}, y_{m-2}\right]\left(\left[x_{m-1}, y_{m-1}\right]^{\epsilon}-\left[x_{m-1}, y_{m-1}\right]\right) \in T_{L}\left(U_{m}\right)$.

Proof Denote $c_{i}:=\left[x_{i}, y_{i}\right]$ for $i=1, \ldots, m-1$ and let $\varphi$ be any substitution in $U_{m}$ such that $\varphi\left(c_{1} \ldots c_{m-2}\right) \neq 0$. Then its value belongs to $\operatorname{span}_{F}\left\langle e_{1 m-1}, e_{2 m}\right\rangle$. Hence just the $(m-1, m)$-entry of $\varphi\left(c_{m-1}\right)$ contributes to $\varphi\left(c_{1} \ldots c_{m-1}\right)$. By the way it is the same entry as $\varphi\left(c_{m-1}^{\epsilon}\right)$, hence $\varphi\left(c_{1} \ldots c_{m-2} c_{m-1}^{\epsilon}-c_{1} \ldots c_{m-2} c_{m-1}\right)=0$.

The polynomials listed so far constitute the candidate set of generators of $T_{L}\left(U_{m}\right)$. Let us fix the notation:

Definition 3.4 Let $\mathscr{I}$ denote the set constituted by the following differential polynomials:

$$
\begin{aligned}
& x^{\epsilon^{2}}-x^{\epsilon}, x^{\delta^{2}}, x^{\delta \epsilon}-x^{\delta}, x^{\epsilon \delta}, \\
& x^{\delta}\left[x_{1}, x_{2}\right],\left[x_{1}, x_{2}\right] x^{\delta},\left[x_{1}^{\delta}, x_{2}\right]+\left[x_{1}, x_{2}^{\delta}\right], x^{\epsilon}\left[x_{1}, x_{2}\right],\left[x_{1}, y_{1}\right] \ldots\left[x_{m}, y_{m}\right], \\
& {\left[x_{1}, y_{1}\right] \ldots\left[x_{m-1}, y_{m-1}\right] x^{\epsilon},\left[x_{1}, y_{1}\right] \ldots\left[x_{m-2}, y_{m-2}\right]\left(\left[x_{m-1}, y_{m-1}\right]^{\epsilon}-\left[x_{m-1}, y_{m-1}\right]\right),}
\end{aligned}
$$

where all indeterminates belong to $X$. Moreover, let $I$ denote the $T_{L}$-ideal generated by $\mathscr{I}$.

The $T_{L}$-ideal $I$ is contained in $T_{L}\left(U_{m}\right)$, and we are going to prove that in fact the reverse inclusion holds as well. As a first step, we are going to determine a set of polynomials spanning $\Gamma_{n}^{L}$ modulo $I$. The second step will be to prove their linear independence modulo $T_{L}\left(U_{m}\right)$, in the next section.

As in [6] let us denote by $\mathscr{B}_{k}$ the subset of the basis of $B_{X}^{L}$ constituted by the products of $k$ nssc's, and let $\Gamma_{n, k}:=\Gamma_{n}^{L} \cap \mathscr{B}_{k}$, for all $k \geqslant 1$. Since the identity $\left[x_{1}, y_{1}\right] \ldots\left[x_{m}, y_{m}\right] \in I$, any product of $k \geqslant m$ normal standard commutators is in $I$. Hence any element of $\Gamma_{n}$ can be written, modulo $I$, as a linear combinations of products of at most $m-1 \mathrm{nsc}$ 's. Next let us prove the following 
Lemma 3.5 Let $u$ be a nsc involving a $\delta$-letter. Then $u c \equiv 0 \equiv c u(\bmod I)$ for all normal commutators $c$.

Proof If $u=x^{\delta}$ then $u y^{\epsilon} \equiv u y^{\delta} \equiv 0(\bmod I)$; also, for any $k \geqslant 1$ and any $\alpha \in\{1, \epsilon, \delta\}$ it holds $x^{\delta}\left[y^{\alpha}, y_{1}, \ldots, y_{k}\right] \equiv 0(\bmod I)$, since it follows from $x^{\delta}\left[x_{1}, x_{2}\right] \in I$ under the substitution $x_{1} \rightarrow\left[y^{\alpha}, y_{1}, \ldots, y_{k-1}\right], x_{2} \rightarrow y_{k}$.

Now let $u=\left[x^{\delta}, z_{1}, \ldots, z_{l}\right]$ and use induction on $l$ : we just proved $u c \equiv 0(\bmod I)$ if $l=0$, so consider $u=\left[x^{\delta}, z_{1}, \ldots, z_{l-1}, z_{l}\right]$ and let $u^{\prime}=\left[x^{\delta}, z_{1}, \ldots, z_{l-1}\right]$. Assume $\left[x^{\delta}, z_{1}, \ldots, z_{l-1}\right] c \in I$ for all nsc's $c$. Then $u c=u^{\prime} z_{l} c-z_{l} u^{\prime} c \equiv u^{\prime} z_{l} c(\bmod I)$. Since $z_{l} c=c z_{l}-\left[c, z_{l}\right]$ it holds $u c \equiv\left(u^{\prime} c\right) z_{l}-u^{\prime}\left[c, z_{l}\right] \equiv 0(\bmod I)$, and we are done.

The case $c u \equiv 0$ is completely analogous.

As a consequence, if $u \in \Gamma_{n, k}$ involves a $\delta$-letter and does not belong to $I$ then $k=1$. We can be more precise:

Lemma 3.6 Let $u \in \Gamma_{n, 1}$ involve a $\delta$-letter. Then $u \equiv\left[x_{n}^{\delta}, x_{1}, \ldots, x_{n-1}\right](\bmod I)$.

Proof The statement is trivially true for $n=1$, and for $n=2$ it follows from $\left[x_{1}, x_{2}\right]^{\delta} \in I$. So assume $n \geqslant 3$ and use induction: let $u=\left[x_{i}^{\delta}, x_{1}, \ldots, x_{n}\right]$ with $i \neq n$. Notice that for all $k \geqslant 1$ it holds $\left[x^{\delta}, y_{1}, \ldots, y_{k}, z\right] \equiv\left[x^{\delta}, y_{1}, \ldots, z, y_{k}\right] \bmod I$. Indeed, since $[\cdot, z]$ is a derivation and $\left[x^{\delta}, y_{1}, \ldots, y_{k}\right]=\left[\left[x^{\delta}, y_{1}, \ldots, y_{k-1}\right], y_{k}\right]$ it holds

$$
\begin{aligned}
{\left[x^{\delta}, y_{1}, \ldots, y_{k, z}\right] } & =\left[\left[x^{\delta}, y_{1}, \ldots, y_{k-1}, z\right], y_{k}\right]+\left[\left[x^{\delta}, y_{1}, \ldots, y_{k-1}\right],\left[y_{k}, z\right]\right] \\
& \equiv\left[x^{\delta}, y_{1}, \ldots, y_{k-1}, z, y_{k}\right](\bmod I)
\end{aligned}
$$

by the previous Lemma. Therefore the letters occurring in $u$ in positions $2, \ldots, n$ can be permuted modulo $I$. Then $u \equiv\left[x_{i}^{\delta}, x_{n}, x_{1}, \ldots, x_{n-1}\right]$. Now the conclusion follows from the fact that $\left[x_{i}^{\delta}, x_{n}\right] \equiv\left[x_{n}^{\delta}, x_{i}\right](\bmod I)$.

There is a partial analogous of Lemma 3.5 holding for $\epsilon$-letters:

Lemma 3.7 Let $u$ be a nsc involving an $\epsilon$-letter. Then for all nsc's $c$ it holds $u c \equiv 0$ $(\bmod I)$.

Proof Just replace $\delta$ by $\epsilon$ in the proof of Lemma 3.5.

Instead, it is not true that $c u \equiv 0$. By the way, if $f \in \mathscr{B}_{k}$ involves an $\epsilon$-letter and is not zero modulo $I$, then $f=c_{1} \ldots c_{k}$ where $c_{1}, \ldots, c_{k-1}$ are ordinary standard commutators and $c_{k}$ involves the only $\epsilon$-letter in $f$. Also, if $k \leqslant m-2$ there is not an analogous of Lemma 3.6 holding for $\epsilon$-letters. Just the case of a product of $m-1$ commutators provides something similar:

Lemma 3.8 Let $w=c_{1} \ldots c_{m-1} \in \Gamma_{n, m-1}$ involve an $\epsilon$-letter. If $w$ is not zero $\bmod I$, and $y_{1}<\cdots<y_{l}<x$ are the indeterminates involved in $c_{m-1}$ then $w \equiv$ $c_{1} \ldots c_{m-2}\left(\left[x^{\epsilon}, y_{1}, \ldots, y_{l}\right]+u\right)(\bmod I)$ for some linear combination $u$ of ordinary standard commutators in $y_{1}, \ldots, y_{l}, x$.

Proof The statement is clearly true (with $u=0$ ) if $l=0$, so assume that $l \geqslant$ 1. The proof is somehow similar to the one of Lemma 3.6: let us set $w_{0}:=$ $c_{1} \ldots c_{m-2}$ and, say, $c_{m-1}=\left[y_{i}^{\epsilon}, y_{1}, \ldots, y_{i-1}, y_{i+1}, \ldots, y_{l}, x\right]$. We may permute the 
indeterminates occurring in positions $2, \ldots, l+1$ in the last commutator by the previous Lemma, and bring $x$ in second position so to get $w \equiv w_{0}\left[y_{i}^{\epsilon}, x, y_{1}, \ldots\right](\bmod I)$. Now we may employ the same trick used in Lemma 3.6 using the identity of Lemma 3.3 to get $w \equiv w_{0}\left[x^{\epsilon}, y_{i}, y_{1}, \ldots\right]+w_{0}\left[x, y_{i}, y_{1}, \ldots\right](\bmod I)$. We can now re-order the indeterminates $y_{1}, \ldots y_{l}$ in $\left[x^{\epsilon}, y_{i}, \ldots\right]$ modulo $I$ getting $\left[x^{\epsilon}, y_{1}, \ldots, y_{l}\right]$. For the second summand, we have to separate two cases: if $i=1$ then $\left[x, y_{1}, \ldots\right]$ is already standard, so $u=\left[x, y_{1}, \ldots, y_{l}\right]$. If instead $i>1$ then $\left[x, y_{i}, y_{1}, \ldots\right]$ is not standard, but we may use Jacobi identity and write $\left[x, y_{i}, y_{1}, \ldots\right]=\left[x, y_{1}, y_{i}, \ldots\right]-\left[y_{i}, y_{1}, x, \ldots\right]$, then rearrange the entries in positions $3, \ldots, l+1$ modulo $I$ to get $u=\left[x, y_{1}, \ldots, y_{l}\right]+\left[y_{i}, y_{1}, \ldots, x\right]$.

Definition 3.9 For any $k \geqslant 1$ let us denote by

- $\quad \mathscr{S}_{n, k}^{1}\left(U_{m}\right)$ the subset of $\Gamma_{n, k}$ of all products $c_{1} \ldots c_{k}$ of ordinary standard commutators;

- $\quad \mathscr{S}_{n, k}^{\epsilon}\left(U_{m}\right)$ the set of all products $c_{1} \ldots c_{k} \in \Gamma_{n, k}$ such that $c_{1}, \ldots, c_{k-1}$ are ordinary standard commutators and $c_{k}$ involves an $\epsilon$-letter in its first (possibly the only one) position, if $k \leqslant m-2$;

- $\quad \mathscr{S}_{n, m-1}^{\epsilon}\left(U_{m}\right)$ the set of products $c_{1} \ldots c_{m-1} \in \Gamma_{n, m-1}$ where $c_{1}, \ldots, c_{m-2}$ are ordinary standard commutators, and if $x$ is the indeterminate with maximum name among those involved in $c_{m-1}$ then $x^{\epsilon}$ is placed in first position (that is: $c_{m-1}=\left[x^{\epsilon}, y_{1}, \ldots, y_{l}\right]$ and $\left.x=\max \left\{x, y_{1}, \ldots, y_{l}\right\}\right)$;

- $\mathscr{S}_{n}^{\delta}\left(U_{m}\right)=\left\{\left[x_{n}^{\delta}, x_{1}, \ldots, x_{n-1}\right]\right\}$.

Moreover, working modulo $T_{L}\left(U_{m}\right)$, let us denote by $\Gamma_{n, k}^{\alpha}\left(U_{m}\right)$ the vector subspace of $\Gamma_{n}^{L}\left(U_{m}\right)$ spanned by $\mathscr{S}_{n, k}^{\alpha}\left(U_{m}\right)$.

Gluing together the parts, let $\mathscr{S}_{n}^{1}\left(U_{m}\right)=\bigcup_{k \leqslant m-1} \mathscr{S}_{n, k}^{1}\left(U_{m}\right)$ be the set of selected ordinary polynomials, $\mathscr{S}_{n}^{\epsilon}\left(U_{m}\right)=\bigcup_{k \leqslant m-1} \mathscr{S}_{n, k}^{\epsilon}\left(U_{m}\right)$ the one of polynomials involving an $\epsilon$-letter and, finally, let $\mathscr{S}_{n}\left(U_{m}\right)=\mathscr{S}_{n}^{1}\left(U_{m}\right) \cup \mathscr{S}_{n}^{\epsilon}\left(U_{m}\right) \cup \mathscr{S}_{n}^{\delta}\left(U_{m}\right)$. Extending the notations to the vectors subspaces they span modulo $T_{L}\left(U_{m}\right)$, we will denote $\Gamma_{n}^{\alpha}\left(U_{m}\right)$ the vector subspace of $\Gamma_{n}^{L}\left(U_{m}\right)$ spanned by $\mathscr{S}_{n}^{\alpha}\left(U_{m}\right)$.

We can now summarize all the previous results stating

Theorem 3.10 $\mathscr{S}_{n}\left(U_{m}\right)$ spans $\Gamma_{n}^{L}$ modulo $I$.

Proof Any basis element $c_{1} \ldots c_{k}$ of $\Gamma_{n}$ is either zero or equivalent to a suitable linear combination of elements in $\mathscr{S}_{n}\left(U_{m}\right)$ modulo $I$, so $\mathscr{S}_{n}\left(U_{m}\right)$ spans $\Gamma_{n}$ modulo $I$, by the preceding results.

Hence it follows

Corollary 3.11 $\mathscr{S}_{n}\left(U_{m}\right)$ spans $\Gamma_{n}^{L}\left(U_{m}\right)$.

Proof Since $\mathscr{S}_{n}\left(U_{m}\right)$ spans $\Gamma_{n}^{L}$ modulo $I$ and $I \subseteq T_{L}\left(U_{m}\right)$, the same holds modulo $T_{L}\left(U_{m}\right)$.

Remark 3.12 The size of the sets of polynomials selected so far depend upon the size $m$. For instance, passing from $U_{m-1}$ to $U_{m}$ we must add further polynomials to the sets $\mathscr{S}_{n}^{1}\left(U_{m-1}\right)$ and $\mathscr{S}_{n}^{\epsilon}\left(U_{m-1}\right)$ to get $\mathscr{S}_{n}^{1}\left(U_{m}\right)$ and $\mathscr{S}_{n}^{\epsilon}\left(U_{m}\right)$, while on the contrary $\mathscr{S}_{n}^{\delta}\left(U_{m-1}\right)=\mathscr{S}_{n}^{\delta}\left(U_{m}\right)=\left\{\left[x_{n}^{\delta}, x_{1}, \ldots, x_{n-1}\right]\right\}$. The codimension sequences we are going 
to compute later in this paper will measure exactly how these sizes vary. There are however a couple of points to notice right now: the first one is that the set $\mathscr{S}_{n}^{1}\left(U_{m}\right)$ constitutes a basis for $\Gamma_{n}\left(U T_{m}(F)\right)$, that is for the ordinary algebra of upper triangular matrices without any derivation (see [9]). For the second one, compare the sets $\mathscr{S}_{n}\left(U_{m-1}\right)$ and $\mathscr{S}_{n}\left(U_{m}\right)$. It turns out that

- $\quad \mathscr{S}_{n}^{1}\left(U_{m}\right)=\mathscr{S}_{n}^{1}\left(U_{m-1}\right) \uplus \mathscr{S}_{n, m-1}^{1}\left(U_{m}\right)$, since $\mathscr{S}_{n, k}^{1}\left(U_{m-1}\right)=\mathscr{S}_{n, k}^{1}\left(U_{m}\right)$ for all $k \leqslant$ $m-2$. To put it differently, just multilinear polynomials involving products of $m-1$ nsc's are missing in $\mathscr{S}_{n}^{1}\left(U_{m-1}\right)$ to get the whole $\mathscr{S}_{n}^{1}\left(U_{m}\right)$;

- $\quad \mathscr{S}_{n, k}^{\epsilon}\left(U_{m-1}\right)=\mathscr{S}_{n, k}^{\epsilon}\left(U_{m}\right)$ for all $k \leqslant m-3$;

- $\quad \mathscr{S}_{n, m-2}^{\epsilon}\left(U_{m-1}\right) \varsubsetneqq \mathscr{S}_{n, m-2}^{\epsilon}\left(U_{m}\right)$. So, we have to add a complementary set $\hat{\mathscr{S}}_{n, m-2}^{\epsilon}\left(U_{m}\right)$ to $\mathscr{S}_{n, m-2}^{\epsilon}\left(U_{m-1}\right)$ to get $\mathscr{S}_{n, m-2}^{\epsilon}\left(U_{m}\right)$;

- moreover, multilinear polynomials involving products of $m-1$ nsc's must be added to $\mathscr{S}_{n}^{\epsilon}\left(U_{m-1}\right)$ in order to get $\mathscr{S}_{n}^{\epsilon}\left(U_{m}\right)$.

- Therefore $\mathscr{S}_{n}^{\epsilon}\left(U_{m}\right)=\mathscr{S}_{n}^{\epsilon}\left(U_{m-1}\right) \uplus \hat{\mathscr{S}}_{n, m-2}^{\epsilon}\left(U_{m}\right) \uplus \mathscr{S}_{n, m-1}^{\epsilon}\left(U_{m}\right)$.

The first and easy examples occur when $m=3$ and $n \geqslant 2$. For instance, in these minimal settings, one has

$$
\begin{aligned}
& -\quad \mathscr{S}_{2,1}^{\epsilon}\left(U_{2}\right)=\left\{\left[x_{2}^{\epsilon}, x_{1}\right]\right\} \\
& -\quad \mathscr{S}_{2,1}^{\epsilon}\left(U_{3}\right)=\left\{\left[x_{2}^{\epsilon}, x_{1}\right],\left[x_{1}^{\epsilon}, x_{2}\right]\right\} .
\end{aligned}
$$

To summarize, one has

$$
\mathscr{S}_{n}\left(U_{m}\right)=\mathscr{S}_{n}\left(U_{m-1}\right) \uplus \hat{\mathscr{S}}_{n, m-2}^{\epsilon}\left(U_{m}\right) \uplus \mathscr{S}_{n, m-1}\left(U_{m}\right), \quad \mathscr{S}_{n}^{1}\left(U_{m}\right)=\mathscr{S}_{n}\left(U T_{m}(F)\right) .
$$

\section{The Linear Indipendence of $\mathscr{S}_{n}\left(U_{m}\right)$ Modulo $T_{L}\left(U_{m}\right)$}

In this section we are going to prove that the spanning set $\mathscr{S}_{n}\left(U_{m}\right)$ is indeed linearly independent modulo $T_{L}\left(U_{m}\right)$. To this aim, starting from a given linear combination of a subset of $\mathscr{S}_{n}\left(U_{m}\right)$ assumed to be in $T_{L}\left(U_{m}\right)$, we will exhibit a suitable substitution (that is: an $L$ homomorphisms from $F\left\langle X^{L}\right\rangle$ to $U_{m}$ defined on $X$ ) vanishing on all involved vectors but one, so that it is linearly independent with the remaining ones and can be deleted, shortening selectively the list until it is empty.

The easiest cancellation is the following:

Lemma 4.1 For any $n \geqslant 1$ the vector $\left[x_{n}^{\delta}, x_{1}, \ldots, x_{n-1}\right]$ is linearly independent with the remaining vectors of $\mathscr{S}_{n}\left(U_{m}\right)$.

Proof Assume that $f=\sum_{u} \alpha_{u} u+\beta\left[x_{n}^{\delta}, x_{1}, \ldots, x_{n-1}\right] \in T_{L}\left(U_{m}\right)$, where $u$ runs in $\mathscr{S}_{n}^{1}\left(U_{m}\right) \cup \mathscr{S}_{n}^{\epsilon}\left(U_{m}\right)$ and $\alpha_{u}, \beta \in F$. Let $\varphi$ be the substitution sending all the letters of $X$ in $e_{11}$. Then $\varphi(f)=0$. By the way, $\varphi(u)=0$ for all $u$, since $e_{11} \in \operatorname{ker}(\epsilon)$ and the ordinary commutators vanish under $\varphi$. Since $e_{11}^{\delta}=e_{1 m}$ and $\left[e_{1 m}, e_{11}, \ldots, e_{11}\right] \neq 0$ it follows $\beta=0$, so $\left[x_{n}^{\delta}, x_{1}, \ldots, x_{n-1}\right]$ is linearly independent modulo $T_{L}\left(U_{m}\right)$ with the remaining vectors of $\mathscr{S}_{n}\left(U_{m}\right)$.

In other words, we just proved that

$$
\Gamma_{n}^{L}\left(U_{m}\right)=\Gamma_{n}^{\delta}\left(U_{m}\right) \oplus\left(\Gamma_{n}^{1}\left(U_{m}\right)+\Gamma_{n}^{\epsilon}\left(U_{m}\right)\right) .
$$


Lemma 4.2 The set $\mathscr{S}_{n}^{1}\left(U_{m-1}\right)$ is a linearly independent subset of $\mathscr{S}_{n}\left(U_{m}\right)$ and

$$
\Gamma_{n}^{1}\left(U_{m}\right)+\Gamma_{n}^{\epsilon}\left(U_{m}\right)=\Gamma_{n}^{1}\left(U_{m-1}\right) \oplus\left(\Gamma_{n, m-1}^{1}\left(U_{m}\right)+\Gamma_{n}^{\epsilon}\left(U_{m}\right)\right) .
$$

Proof Recall that $\mathscr{S}_{n}^{1}\left(U_{m-1}\right)=\bigcup_{k \leqslant m-2} \mathscr{S}_{n, k}^{1}\left(U_{m}\right)$, and assume

$$
f=\sum_{u \in \mathscr{S}_{n}^{1}\left(U_{m-1}\right)} \alpha_{u} u+\sum_{v \in \mathscr{S}_{n, m-1}^{1}\left(U_{m}\right)} \beta_{v} v+\sum_{w \in \mathscr{S}_{n}^{\epsilon}\left(U_{m}\right)} \gamma_{w} w \equiv 0 \quad\left(\bmod T_{L}\left(U_{m}\right)\right) .
$$

Let $K$ the subalgebra of $U_{m}$ having null last $m$-th column. Then $K \cong U T_{m-1}(F)$ as $F$ algebras. Any evaluation $\varphi: X \rightarrow K$ vanishes on $\mathscr{S}_{n}^{\epsilon}\left(U_{m}\right)$ because $K \subseteq \operatorname{ker}(\epsilon)$, as well as any product of $m-1$ commutators, hence $0=\varphi(f)=\varphi\left(\sum_{u} \alpha_{u} u\right)$ for all such substitution. This means that $\sum_{u} \alpha_{u} u$ is a (ordinary) polynomial identity for $U T_{m-1}(F)$. By the way, the set $\mathscr{S}_{n}^{1}\left(U_{m-1}\right)$ gives rise to a basis for $\Gamma_{n}\left(U_{m-1}\right)$, hence all $\alpha_{u}$ 's are zero.

So the problem is confined to prove the linear independence of the vectors of $\mathscr{S}_{n, m-1}^{1}\left(U_{m}\right) \cup \mathscr{S}_{n}^{\epsilon}\left(U_{m}\right)$. As a first step, for any fixed $1 \leqslant k \leqslant m-1$ let us consider the sets

$$
\begin{aligned}
& \Lambda_{n, k}:=\left\{\left(l_{1}, \ldots, l_{k}\right) \mid l_{1}, \ldots, l_{k-1} \geqslant 2, l_{k} \geqslant 1, l_{1}+\cdots+l_{k}=n\right\} \\
& \Lambda_{n, k}^{\prime}:=\left\{\left(l_{1}, \ldots, l_{k}\right) \mid l_{1}, \ldots, l_{k-1}, l_{k} \geqslant 2, l_{1}+\cdots+l_{k}=n\right\} .
\end{aligned}
$$

$\Lambda_{n, k}\left(\Lambda_{n, k}^{\prime}\right.$, respectively) is simply the set of all compositions of the integer $n$ in $k$ parts satisfying the inequalities $l_{k} \geqslant 1$ and the other ones $\geqslant 2$ ( $l_{i} \geqslant 2$ for all $i$ 's, respectively). Of course, $\Lambda_{n, k}^{\prime} \subseteq \Lambda_{n, k}$. Consider on $\Lambda_{n, k}$ the right lexicographic order, that is $\left(l_{1}, \ldots, l_{k}\right)<$ $\left(m_{1}, \ldots, m_{k}\right)$ if $l_{k}=m_{k}, \ldots, l_{i+1}=m_{i+1}$ and $l_{i}<m_{i}$ for some $1 \leqslant i \leqslant k$.

Definition 4.3 Let $w=c_{1} \ldots c_{k} \in \mathscr{S}_{n, k}$. The word $\|w\|:=z_{1} \ldots z_{k}$ obtained taking the first letter $z_{i}$ of $c_{i}$ will be called the main word of $w$. The letter $z_{i}$ will be called the main letter of $c_{i}$. The other letters of $c_{i}$ will be called secondary letters of $c_{i}$. The sequence $l(w):=\left(l_{1}, \ldots, l_{k}\right)$ of the lengths of the commutators $c_{1}, \ldots, c_{k}$ will be called the structure of $w$.

So, for instance, the vector $w=\left[x_{2}, x_{1}, x_{4}\right]\left[x_{7}, x_{5}\right]\left[x_{3}^{\epsilon}, x_{6}\right] \in \mathscr{S}_{7,3}\left(U_{5}\right)$ has $\|w\|=$ $x_{2} x_{7} x_{3}^{\epsilon}$ and structure $l(w)=(3,2,2)$. Notice that $w \notin \mathscr{S}_{7,3}\left(U_{4}\right)$. Notice also that for any $w \in \mathscr{S}_{n, k}\left(U_{m}\right)$ the structure $l(w)$ belongs to $\Lambda_{n, k}$.

Now we are going to delete all polynomials of $\mathscr{S}_{n, k}^{\epsilon}\left(U_{m}\right)$ for $k \leqslant m-2$ from the list, one by one. To make the clearest possible computations, we need an abuse of notation. For any $k \leqslant m-2$ let $\theta_{k}: U_{k} \rightarrow U_{m}$ be the map defined by $\theta_{k}\left(e_{i j}\right)=e_{i+m-k j+m-k}$. Its image is the subalgebra $A_{k}$ of $U_{m}$ of all matrices whose rows $1, \ldots, m-k$ are zero. It is immediate to notice that $\theta_{k}$ is not only an algebra isomorphism beween $U_{k}$ and $A_{k}$, but it commutes with the $L$-action: for any $u \in U_{k}$ one has $\theta_{k}\left(\left[u, e_{k k}\right]\right)=\theta_{k}(u)^{\epsilon}$ and, similarly, $\theta_{k}\left(\left[u, e_{1 k}\right]\right)=\theta_{k}(u)^{\delta}$. In what follows, by $A_{k}$-substitution $\varphi$ sending $x \rightarrow a \in U_{k}$ we mean the substitution sending $x \in X$ to $\theta_{k}(a) \in A_{k}$, abusing the notation merely in order to simplify the computations.

Example 4.4 Let $f=\left[x_{3}, x_{1}, x_{2}\right]\left[x_{4}^{\epsilon}, x_{5}\right]$ and suppose we need to exhibit a nonzero evaluation of $f$ in the small $3 \times 3$-lower corner $A_{3}$ of $U_{m}$. We should write, for instance, $x_{1}, x_{2} \rightarrow e_{m-2 m-2}, x_{3} \rightarrow e_{m-1 m-2}, x_{4} \rightarrow e_{m-1 m}$ and $x_{5} \rightarrow e_{m m}$. We shall instead employ the $A_{3}$-substitution sending $x_{1}, x_{2} \rightarrow e_{22}, x_{3} \rightarrow e_{12}, x_{4} \rightarrow e_{23}$ and $x_{5} \rightarrow e_{33}$, that is evaluate the polynomial inside $U_{3}$ and just then going back inside $A_{3} \subseteq U_{m}$. 
Notice also that the embeddings $\theta_{k}$ provide a canonical ascending chain $U_{2} \subseteq U_{3} \subseteq$ $\cdots \subseteq U_{m} \subseteq \ldots$, and hence the descending chain $T_{L}\left(U_{2}\right) \supseteq T_{L}\left(U_{3}\right) \supseteq \cdots \supseteq T_{L}\left(U_{m}\right) \supseteq$ .. of $T_{L}$-ideals of $F\left\langle X^{L}\right\rangle$.

Definition 4.5 Let $1 \leqslant k \leqslant m-2$ and let $w=c_{1} \ldots c_{k} \in \mathscr{S}_{n, k}\left(U_{m}\right)$. We define $\varphi_{w}$ to be the $A_{k+1}$-substitution sending the main letter of $c_{i}$ in $e_{i i+1}$ and the secondary letters of $c_{i}$ in $e_{i+1 i+1}$.

Clearly, $\varphi_{w}$ is designed to have $\varphi_{w}(w) \neq 0$, and indeed it holds $\varphi_{w}(w)=e_{1 k+1} \in U_{k+1}$, so that it actually evalutates to $e_{m-k m} \in A_{k+1}$. Besides, we have

Lemma 4.6 Let $w \in \mathscr{S}_{n, k}^{\epsilon}\left(U_{m}\right)$ for $k \leqslant m-2$, and let $\varphi_{w}$ be its associated $A_{k+1}$ substitution. Then

1. for any $h>k$, for any $u \in \mathscr{S}_{n, h}^{\epsilon}\left(U_{m}\right)$ it holds $\varphi_{w}(u)=0$;

2. if $u \in \mathscr{S}_{n, k}^{\epsilon}\left(U_{m}\right)$ and $\varphi_{w}(u) \neq 0$ then $l(u) \geqslant l(w)$. Moreover, if $l(u)=l(w)$ then $u=w$.

\section{Proof}

1. Any product of more than $k$ commutators is an identity for $U_{k+1}$, so $\varphi_{w}(u)=0$.

2. Let $u=d_{1} \ldots d_{k} \in \mathscr{S}_{n, k}^{\epsilon}\left(U_{m}\right)$ and assume that $\varphi_{w}(u) \neq 0$. Just $k$ among the letters $x_{1}, \ldots, x_{n}$ are substituted by off-diagonal elements, and diagonal matrices commute. So, by the pigeonhole principle, each $d_{i}$ must involve exactly one among the main letters $z_{1}, \ldots, z_{k}$ of $w$. Actually, since any non-identity permutation of the factors in $e_{12} \ldots e_{k k+1}$ causes the product to vanish, the letter $z_{i}$ occurs in $d_{i}$ for all $i$, and it must occur in its first or second position, otherwise $\varphi_{w}\left(d_{i}\right)=0$.

Now notice that if a secondary letter of $c_{k}$ occurs in $d_{i}$ for $i<k$ then $\varphi_{w}(u)=0$ : exactly one among the entries of $\varphi\left(d_{i}\right)$ is $e_{i i+1}$, and if $e_{k+1 k+1}$ occurs in it then $\varphi\left(d_{i}\right)=$ 0 because $\left[e_{i i+1}, e_{k+1 k+1}\right]=0$. Therefore all the letters of $c_{k}$ are confined within $d_{k}$, derived or not. Hence $l\left(d_{k}\right) \geqslant l\left(c_{k}\right)$. Therefore if $l\left(d_{k}\right)>l\left(c_{k}\right)$ then $l(u)>l(w)$. If instead $l\left(d_{k}\right)=l\left(c_{k}\right)$ then $d_{k}$ and $c_{k}$ must involve the same letters. Since however $z_{k}$ must be an $\epsilon$-letter occurring in $d_{k}$, it has to be placed in first position, so $d_{k}=c_{k}$. If $k=1$ the task is done, so assume $k>1$ and let us compare $d_{k-1}$ and $c_{k-1}$.

As before, no secondary letter of $c_{k-1}$ may appear in $d_{i}$ if $i<k-1$, so $l\left(d_{k-1}\right) \geqslant$ $l\left(c_{k-1}\right)$ and, if the inequality is strict, this means $l(u)>l(w)$. If instead $l\left(d_{k-1}\right)=$ $l\left(c_{k-1}\right)$ then $c_{k-1}$ and $d_{k-1}$ involve exactly the same letters, and $z_{k-1}$ is placed in the first or second position of $d_{k-1}$. By the way, since the sets of letters occurring in $c_{k-1}$ and $d_{k-1}$ are the same, they have the same minimum, and this letter must be placed in position 2 both in $d_{k-1}$ and in $c_{k-1}$. Hence $z_{k-1}$ must be in the first position of $d_{k-1}$, hence $d_{k-1}=c_{k-1}$.

These arguments apply to the remaining commutators of $u$, so either $l(u)>l(w)$ or $l(u)=l(w)$. In the latter case the previous arguments show that then $d_{i}=c_{i}$ for all $i$, hence $u=w$.

Then we get a simple algorithm to delete all the vectors in $\mathscr{S}_{n, k}^{\epsilon}\left(U_{m}\right)$ : for increasing $k$ 's, take any element $w$ in $\mathscr{S}_{n, k}^{\epsilon}\left(U_{m}\right)$ of maximum structure $l=\left(l_{1}, \ldots, l_{k}\right)$ and consider the associated $A_{k}$-substitution $\varphi_{w}$. Then $w$ is the only element of $\mathscr{S}_{n, k}^{\epsilon}\left(U_{m}\right)$ not vanishing under $\varphi_{w}$, hence it is linearly independent with all the vectors not yet cancelled. Then we may delete $w$ from the list and repeat the process until no vector of $\mathscr{S}_{n, m-2}^{\epsilon}\left(U_{m}\right)$ remains. Then 
we have to stop: this procedure does not work with elements of $\mathscr{S}_{n, m-1}^{1}\left(U_{m}\right) \cup \mathscr{S}_{n, m-1}^{\epsilon}\left(U_{m}\right)$, essentially because $e_{m-1 m}^{\epsilon}=e_{m-1 m}$ and the last main letter of a vector may be either ordinary or differential without affecting the evaluation. Therefore so far we obtained

$$
\Gamma_{n}^{L}\left(U_{m}\right)=\Gamma_{n}^{\delta}\left(U_{m}\right) \oplus \bigoplus_{k=1}^{m-2} \Gamma_{n, k}^{1}\left(U_{m}\right) \oplus \bigoplus_{k=1}^{m-2} \Gamma_{n, k}^{\epsilon}\left(U_{m}\right) \oplus\left(\Gamma_{n, m-1}^{1}\left(U_{m}\right)+\Gamma_{n, m-1}^{\epsilon}\left(U_{m}\right)\right)
$$

Definition 4.7 If $w=c_{1} \ldots c_{m-1} \in \mathscr{S}_{n, m-1}^{\epsilon}\left(U_{m}\right)$ let $\psi_{w}$ be the substitution sending, for each $1 \leqslant i \leqslant m-2$, the main letter of $c_{i}$ in $e_{i i+1}$ and all its secondary letters in $e_{i+1 i+1}$, and then sending the remaining letters of $\left\{x_{1}, \ldots, x_{n}\right\}$ in $e_{m-1 m}+e_{m m}$.

Clearly $\psi_{w}(w) \neq 0$, and we have

Lemma 4.8 If $u \in \mathscr{S}_{n, m-1}^{\epsilon}\left(U_{m}\right)$ and $\psi_{w}(u) \neq 0$ then $l(u) \geqslant l(w)$. If the equality holds, then $u=w$.

Proof Let $u=d_{1} \ldots d_{m-1} \in \mathscr{S}_{n, m-1}\left(U_{m}\right)$ satisfy $\psi_{w}(u) \neq 0$. Then all the letters of $u$ whose names occur in $c_{m-1}$ are confined within $d_{m-1}$. Indeed, if any of them occurs in some $d_{i}$ with $i<m-1$ then it may happen $\psi_{w}\left(d_{i}\right) \neq 0$, but necessarily $\psi_{w}\left(d_{i}\right) \psi_{w}\left(d_{i+1}\right)=0$, since $\psi_{w}\left(d_{i}\right)=e_{j m}$ for some $j$ and $\psi_{w}\left(d_{i+1}\right) \neq e_{m m}$, the only choice not annihilating the product. In particular, $l\left(d_{m-1}\right) \geqslant l\left(c_{m-1}\right)$. If the equality holds then the names of the letters of $c_{m-1}$ and $d_{m-1}$ form the same set, and since $\psi_{w}\left(d_{m-1}\right) \neq 0$ the commutator $d_{m-1}$ cannot be ordinary. Thus an $\epsilon$-letter must occur in it, and therefore this letter must be the one with maximum name among them, so the same $\epsilon$-letter of $c_{m-1}$. Hence $d_{m-1}=c_{m-1}$.

Now take a look at $u_{0}=d_{1} \ldots d_{m-2}$. It is a product of $m-2$ ordinary commutators in the remaining $n-l\left(c_{m-1}\right)$ letters, and just $m-2$ of them have been specialized in non diagonal elements $e_{i i+1}$. Then the same arguments employed in the proof of Lemma 4.6 apply, so either $l(u)>l(w)$ or $u_{0}=c_{1} \ldots c_{m-2}$.

As a consequence, we get

Proposition 4.9 The set $\mathscr{S}_{n, m-1}\left(U_{m}\right)$ is linearly independent modulo $T_{L}\left(U_{m}\right)$.

Proof Choose any $\epsilon$-element $w \in \mathscr{S}:=\mathscr{S}_{n, m-1}^{1}\left(U_{m}\right) \cup \mathscr{S}_{n, m-1}^{\epsilon}\left(U_{m}\right)$ of maximal structure $l=\left(l_{1}, \ldots, l_{m-1}\right)$ and consider the associated substitution $\psi_{w}$. By the previous Lemma $w$ is the only element in $\mathscr{S}$ not vanishing under $\psi_{w}$. Hence $w$ is linearly independent with the remaining elements of $\mathscr{S}$, and can be deleted. Replacing $\mathscr{S}$ by $\mathscr{S} \backslash\{w\}$, we repeat the process until no $\epsilon$-vector remains in $\mathscr{S}$. Then $\mathscr{S}=\mathscr{S}_{n, m-1}^{1}$ is part of a basis of $\Gamma_{n}\left(U T_{m}(F)\right)$ modulo $T\left(U T_{m}(F)\right)$, so it is linearly independent modulo $T_{L}\left(U_{m}\right)$ too.

Summing up all the previous results, we get the main result

Theorem 4.10 The set $\mathscr{S}_{n}\left(U_{m}\right)$ is an F-basis of the vector space $\Gamma_{n}^{L}\left(U_{m}\right)$. Hence $I=$ $T_{L}\left(U_{m}\right)$.

An equivalent, more comprehensive form for the results so far obtained is

Theorem 4.11 Let $\mathscr{I}$ be the set of differential polynomials of Definition 3.4. Then $\mathscr{I}$ generates all the differential polynomial identities of $U_{m}$. Moreover, the cosets of the polynomials in $\mathscr{S}_{n}\left(U_{m}\right)$ form an $F$-basis of the factor space $\Gamma_{n}^{L}\left(U_{m}\right)=\Gamma_{n}^{L} /\left(\Gamma_{n}^{L} \cap T_{L}\left(U_{m}\right)\right)$. 


\section{Differential Cocharacter Sequence of $U_{m}$}

The set $\Gamma_{n}^{L}\left(U_{m}\right)$ is not just a vector subspace of $P_{n}^{L}\left(U_{m}\right)$ : as already mentioned in Section 2, the natural action of the symmetric group $S_{n}$ defined by $\sigma x_{i}^{\alpha}=x_{\sigma(i)}^{\alpha}$ for all $i \in\{1, \ldots, n\}$, $\sigma \in S_{n}$ and $\alpha \in\{1, \delta, \epsilon\}$, turns $\Gamma_{n}^{L}\left(U_{m}\right)$ into a left $S_{n}$-module. In this section we are going to describe its $S_{n}$-structure.

Recall that a complete set of representatives for the isomorphism classes of irreducible $S_{n}$-modules is in a bijective correspondence with the set of partitions of $n$. If $\lambda \vdash n$ is one of them, we shall abuse the notation and use the same symbol $\lambda$ to mean a (fixed) irreducible $S_{n}$-module in the isomorphism class indexed by $\lambda$. We shall denote $\lambda=\llbracket \lambda_{1}, \ldots, \lambda_{k} \rrbracket$ the partition $\lambda$ with parts $\lambda_{1} \geqslant \lambda_{2} \geqslant \cdots \geqslant \lambda_{k}$.

At first, let us study the $S_{n}$-proper cocharacters of $U_{2}$, that is the $S_{n}$-structure of $\Gamma_{n}\left(U_{2}\right)$.

Proposition 5.1 The structure of $\Gamma_{n}^{L}\left(U_{2}\right)$ is the following:

- $\Gamma_{0}^{L}\left(U_{2}\right) \cong F$;

- $\quad \Gamma_{1}^{L}\left(U_{2}\right) \cong 2 \cdot \llbracket 1 \rrbracket$;

- for $n \geqslant 2$ it holds $\Gamma_{n}^{L}\left(U_{2}\right) \cong \llbracket n-1,1 \rrbracket \oplus 2 \cdot \llbracket n \rrbracket$.

Proof If $n=0$ the only proper polynomial of degree 0 is $1_{F}$, so $\Gamma_{0}^{L}\left(U_{2}\right)=F$. The case $n=1$ is almost as trivial: $\Gamma_{1}^{L}\left(U_{2}\right)=F x_{1}^{\delta} \oplus F x_{1}^{\epsilon}$, and each summand is isomorphic to the $S_{1}$ -

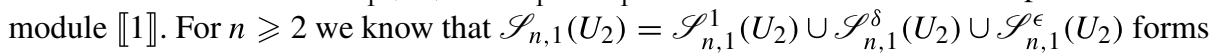
a basis for $\Gamma_{n}^{L}\left(U_{2}\right)$. In fact for $\alpha \in\{1, \delta, \epsilon\}$, each $\Gamma_{n, 1}^{\alpha}\left(U_{2}\right)$ is a submodule of $\Gamma_{n}^{L}\left(U_{2}\right)$, and $\Gamma_{n, 1}^{1}\left(U_{2}\right) \cong \llbracket n-1,1 \rrbracket, \Gamma_{n, 1}^{\delta}\left(U_{2}\right) \cong \Gamma_{n, 1}^{\epsilon}\left(U_{2}\right) \cong \llbracket n \rrbracket$. Therefore $\Gamma_{n}^{L}\left(U_{2}\right) \cong \llbracket n-1,1 \rrbracket \oplus 2$. $\llbracket n \rrbracket$.

Notice that the first summand $\llbracket n-1,1 \rrbracket$ of $\Gamma_{n}^{L}\left(U_{2}\right)$ is precisely the ordinary proper $n$-th cocharacter of $\Gamma_{n}\left(U T_{2}(F)\right)$. The same holds for any $m \geqslant 2$ and, more generally, for all $n$ and $m$ the spaces $\Gamma_{n}^{1}\left(U_{m}\right), \Gamma_{n}^{\delta}\left(U_{m}\right)$ and $\Gamma_{n}^{\epsilon}\left(U_{m}\right)$ are $S_{n}$-submodules of $\Gamma_{n}^{L}\left(U_{m}\right)$.

Remark 5.2 Clearly the decompositions

- $\quad \Gamma_{0}^{L}\left(U_{m}\right)=F$

- $\Gamma_{1}^{L}\left(U_{m}\right)=2 \cdot \llbracket 1 \rrbracket$

hold for all $m \geqslant 2$. Then we pass to study the $\Gamma_{n}^{\alpha}\left(U_{m}\right)$ 's for $n \geqslant 2$.

It is easy to see that

Lemma 5.3 For all $n \geqslant 2$ it holds $\Gamma_{n}^{\delta}\left(U_{m}\right) \cong_{S_{n}} \llbracket n \rrbracket$.

Indeed $\Gamma_{n}^{\delta}\left(U_{m}\right)$ is spanned by the only vector $\left[x_{n}^{\delta}, x_{1}, \ldots, x_{n-1}\right]$ and $S_{n}$ acts trivially on it, modulo $T_{L}\left(U_{m}\right)$. Notice also that $\Gamma_{n}^{\delta}\left(U_{m}\right) \cong \Gamma_{n}^{\delta}\left(U_{m-1}\right)$.

Now, for the general $m \geqslant 3$, we may recursively describe $\Gamma_{n}^{1}\left(U_{m}\right)$ and $\Gamma_{n}^{\epsilon}\left(U_{m}\right)$ by means of $\Gamma_{n}^{1}\left(U_{m-1}\right)$ and $\Gamma_{n}^{\epsilon}\left(U_{m-1}\right)$. The description of $\Gamma_{n}^{1}\left(U_{m}\right)$ is actually already known (see [9]):

Lemma 5.4 For all $n \geqslant 2$ it holds

$$
\begin{gathered}
\Gamma_{n}^{1}\left(U_{m}\right) \cong S_{n} \Gamma_{n}^{1}\left(U_{m-1}\right) \oplus \bigoplus_{l \in \Lambda_{n, m-1}^{\prime}}\left(\llbracket l_{1}-1,1 \rrbracket \otimes \cdots \otimes \llbracket l_{m-1}-1,1 \rrbracket\right)^{S_{n}} \\
\cong_{S_{n}} \Gamma_{n}\left(U T_{m}(F)\right) .
\end{gathered}
$$


The explicit decomposition of $\Gamma_{n}^{1}\left(U_{m}\right)$ is then the following

Corollary 5.5 For all $n \geqslant 2$ it holds

$$
\Gamma_{n}^{1}\left(U_{m}\right) \cong S_{n} \bigoplus_{k=1}^{m-1} \bigoplus_{l \in \Lambda_{n, k}^{\prime}}\left(\llbracket l_{1}-1,1 \rrbracket \otimes \cdots \otimes \llbracket l_{k}-1,1 \rrbracket\right)^{S_{n}} .
$$

The decomposition of $\Gamma_{n}^{\epsilon}\left(U_{m}\right)$ deserves some care

Proposition 5.6 Let $m \geqslant 3$. Then for all $n \geqslant 2$ it holds

$$
\begin{gathered}
\left.\Gamma_{n}^{\epsilon}\left(U_{m}\right) \cong S_{n} \Gamma_{n}^{\epsilon}\left(U_{m-1}\right) \oplus \bigoplus_{l \in \Lambda_{n, m-2}^{\prime}}\left(\llbracket l_{1}-1,1 \rrbracket \otimes \cdots \otimes \llbracket l_{m-2}-1,1\right) \rrbracket\right)^{S_{n}} \\
\oplus \bigoplus_{l \in \Lambda_{n, m-1}}\left(\llbracket l_{1}-1,1 \rrbracket \otimes \cdots \otimes \llbracket l_{m-2}-1,1 \rrbracket \otimes \llbracket l_{m-1} \rrbracket\right)^{S_{n}}
\end{gathered}
$$

Proof Let us work modulo $T_{L}\left(U_{m}\right)$; accordingly, we will simply write $w$ instead of $w+T_{L}\left(U_{m}\right)$, and talk of linear independence, basis and so on without specifying modulo $T_{L}\left(U_{m}\right)$. With obvious significance, consider the set partition

$$
\mathscr{S}_{n}^{\epsilon}\left(U_{m}\right)=\mathscr{S}_{n, \leqslant m-3}^{\epsilon}\left(U_{m}\right) \uplus \mathscr{S}_{n, m-2}^{\epsilon}\left(U_{m}\right) \uplus \mathscr{S}_{n, m-1}^{\epsilon}\left(U_{m}\right) .
$$

As already remarked it holds $\mathscr{S}_{n, \leqslant m-3}^{\epsilon}\left(U_{m}\right)=\mathscr{S}_{n, \leqslant m-3}^{\epsilon}\left(U_{m-1}\right)$, and we will denote $W_{\leqslant m-3}$ the vector space spanned by this set. In the same fashion define $W_{m-2}$ and $W_{m-1}$, hence $\Gamma_{n}^{\epsilon}\left(U_{m}\right)=W_{\leqslant m-3} \oplus W_{m-2} \oplus W_{m-1}$. While the whole vector space is an $S_{n}$-module, just the last summand is an $S_{n}$-submodule of it, and we are going to determine its structure first.

A finer partition of $\mathscr{S}_{n, m-1}^{\epsilon}\left(U_{m}\right)$ is indexed by the structures $l \in \Lambda_{n, m-1}$, that is by the $(m-1)$-tuples $\left(l_{1}, \ldots, l_{m-1}\right)$ such that $l_{m-1} \geqslant 1, l_{i} \geqslant 2$ for other $i$, and $l_{1}+\cdots+l_{m-1}=n$. Then

$$
\mathscr{S}_{n, m-1}^{\epsilon}\left(U_{m}\right)=\biguplus_{l \in \Lambda_{n, m-1}} \mathscr{S}_{n, m-1}^{\epsilon}\left(U_{m}, l\right) .
$$

Let us denote $W_{m-1}(l)=\operatorname{span}_{F}\left\langle\mathscr{S}_{n, m-1}\left(U_{m}, l\right)\right\rangle$ for any $l \in \Lambda_{n, m-1}$. Then it is easy to see that $W_{m-1}=\bigoplus_{l \in \Lambda_{n, m-1}} W_{m-1}(l)$ is a (inner) $S_{n}$-module decomposition. Moreover, each $W_{m-1}(l)$ is cyclic, generated by any element of $\mathscr{S}_{n, m-1}^{\epsilon}\left(U_{m}, l\right)$. Indeed, let $w \in \mathscr{S}_{n, m-1}^{\epsilon}\left(U_{m}\right)$, namely $w=c_{1} \ldots c_{m}$ with structure $l=l(w)$, and let $L_{i}$ be the set of names involved in $c_{i}$. Recall that if $\left\|c_{m-1}\right\|=x^{\epsilon}$ then the name of $x$ is the maximum of $L_{m-1}$. Let us denote by $S_{L}$ the symmetric group on a set $L$ of symbols, and define

$$
H_{w}:=S_{L_{1}} \times S_{L_{2}} \times \cdots \times S_{L_{m-1}} \leqslant S_{n}
$$

Then $H_{w}$ is isomorphic to the direct (external) product $S_{l_{1}} \times \cdots \times S_{l_{m-1}}$, and $F H_{w} w$ is an $H_{w}$-module clearly isomorphic to $\llbracket l_{1}-1,1 \rrbracket \otimes \cdots \otimes \llbracket l_{m-2}-1,1 \rrbracket \otimes \llbracket l_{m-1} \rrbracket$. Inducing in $S_{n}$, we get precisely $W_{m-1}(l)$ : each vector in $\mathscr{S}_{n, m-1}^{\epsilon}\left(U_{m}, l\right)$ is in it, and it is easy to find out that $\operatorname{dim}\left(F H_{w} w\right)^{S_{n}}=\left|\mathscr{S}_{n, m-1}^{\epsilon}\left(U_{m}, l\right)\right|$, so they are the same module. Therefore

$$
W_{m-1} \cong S_{n} \bigoplus_{l \in \Lambda_{m, n-1}}\left(\llbracket l_{1}-1,1 \rrbracket \otimes \cdots \otimes \llbracket l_{m-2}-1,1 \rrbracket \otimes \llbracket l_{m-1} \rrbracket\right)^{S_{n}} .
$$


Our next step is to consider the vector space $W_{m-2}$. It is not an $S_{n}$-submodule of $\Gamma_{n}^{\epsilon}\left(U_{m}\right)$; nevertheless, the cosets of its elements constitute a submodule of the factor $S_{n}$-module $\Gamma_{n}^{\epsilon}\left(U_{m}\right) / W_{m-1}$. Denote it by $\overline{W_{m-2}}$, and let $w=c_{1} \ldots c_{m-2} \in \mathscr{S}_{n, m-2}^{\epsilon}\left(U_{m}\right)$ be of structure $l=\left(l_{1}, \ldots, l_{m-2}\right) \in \Lambda_{n, m-2}$. As before the coset $\bar{w}$ generates an $H_{w}$-module, whose induced $S_{n}$-module is the whole $\overline{W_{m-2}(l)}$, so we need its $H_{w}$-module structure. Let us distinguish two cases: $l_{m-2}=1$ or $l_{m-2} \geqslant 2$.

If $l_{m-2}=1$ then $F H_{w} w \cong_{H_{w}} \llbracket l_{1}-1,1 \rrbracket \otimes \cdots \otimes \llbracket l_{m-3}-1,1 \rrbracket \otimes \llbracket 1 \rrbracket$, since $S_{1}$ acts trivially on the only $\epsilon$-letter, and we are done. So assume $l_{m-2} \geqslant 2$ and let $\left\|c_{m-2}\right\|=x_{j}^{\epsilon}$. The name $j$ of $x$ is no longer the greatest in $L_{m-2}$, but we may consider the subgroup $S_{\{j\}} \times S_{L_{m-2} \backslash\{j\}}$ of $S_{L_{m-2}}$, acting trivially on the last commutator of $\bar{w}$, so $\bar{w}$ generates a $S_{L_{1}} \times \cdots \times S_{L_{m-3}} \times S_{\{j\}} \times S_{L_{m-2} \backslash\{j\}}$-module isomorphic to $\llbracket l_{1}-1,1 \rrbracket \otimes \cdots \otimes \llbracket l_{m-3}-1,1 \rrbracket \otimes$ $\llbracket 1 \rrbracket \otimes \llbracket l_{m-2}-1 \rrbracket$. Then, inducing this module to $H_{w}$, we get

$$
\begin{aligned}
F H_{w} \bar{w} & \cong H_{w} \\
& \cong l_{1}-1,1 \rrbracket \otimes \cdots \otimes \llbracket l_{m-3}-1,1 \rrbracket \otimes\left(\llbracket 1 \rrbracket \otimes \llbracket l_{m-2}-1 \rrbracket\right)^{S_{L_{m-2}}} \\
& \oplus\left(\llbracket l_{1}-1,1 \rrbracket \otimes \cdots \otimes \llbracket l_{m-3}-1,1 \rrbracket \otimes \llbracket l_{m-2} \rrbracket\right) \\
& \left(\llbracket l_{1}-1,1 \rrbracket \otimes \cdots \otimes \llbracket l_{m-3}-1,1 \rrbracket \otimes \llbracket l_{m-2}-1,1 \rrbracket\right)
\end{aligned}
$$

Finally, let us investigate $W_{\leqslant m-3}$ : as before, it is not an $S_{n}$-submodule of $\Gamma_{n}^{\epsilon}\left(U_{m}\right)$ nor of $\Gamma_{n}^{\epsilon}\left(U_{m}\right) / W_{m-1}$. By the way, it induces an $S_{n}$-submodule $\overline{\overline{W_{\leqslant m-3}}}$ of the factor module $\Gamma_{n}^{\epsilon}\left(U_{m}\right) /\left(W_{m-2} \oplus W_{m-1}\right)$, with basis $\mathscr{S}_{n, \leqslant m-3}^{\epsilon}\left(U_{m}\right)$. As already noticed, actually $\mathscr{S}_{n, \leqslant m-3}^{\epsilon}\left(U_{m}\right)=\mathscr{S}_{n, \leqslant m-3}^{\epsilon}\left(U_{m-1}\right)$, and the $S_{n}$-action on $W_{\leqslant m-3}\left(U_{m}\right)$ modulo $\left(W_{m-2} \oplus\right.$ $\left.W_{m-1}\right) \cap T_{L}\left(U_{m}\right)$ is the same as the one on $W_{\leqslant m-3}\left(U_{m-1}\right)$ modulo $W_{m-2} \cap T_{L}\left(U_{m-1}\right)$.

Hence, by complete reducibility, we get

$$
\Gamma_{n}^{\epsilon}\left(U_{m}\right) \cong S_{n} W_{\leqslant m-3}^{\prime}\left(U_{m}\right) \oplus W_{m-2}^{\prime}\left(U_{m}\right) \oplus W_{m-1},
$$

where $W_{m-2}^{\prime} \cong_{S_{n}} \overline{W_{m-2}}$ and $W_{\leqslant m-3}^{\prime}\left(U_{m}\right) \cong_{S_{n}} \overline{\overline{W_{\leqslant m-3}}}$. We may relate the $S_{n}$-structure of $\Gamma_{n}^{\epsilon}\left(U_{m}\right)$ to the one of $\Gamma_{n}^{\epsilon}\left(U_{m-1}\right)$ inductively: indeed

$$
W_{m-2}^{\prime}\left(U_{m}\right) \cong W_{m-2}\left(U_{m-1}\right) \oplus \bigoplus_{l \in \Lambda_{n, m-2}^{\prime}}\left(\llbracket l_{1}-1,1 \rrbracket \otimes \cdots \otimes \llbracket l_{m-2}-1,1 \rrbracket\right)^{S_{n}},
$$

while $W_{\leqslant m-3}^{\prime}\left(U_{m}\right) \cong{ }_{S_{n}} W_{\leqslant m-3}^{\prime}\left(U_{m-1}\right)$, and in the end we get

$$
\begin{gathered}
\Gamma_{n}^{\epsilon}\left(U_{m}\right) \cong_{S_{n}} \Gamma_{n}^{\epsilon}\left(U_{m-1}\right) \oplus \bigoplus_{l \in \Lambda_{n, m-2}^{\prime}}\left(\llbracket l_{1}-1,1 \rrbracket \otimes \cdots \otimes \llbracket l_{m-2}-1,1 \rrbracket\right)^{S_{n}} \\
\oplus \bigoplus_{l \in \Lambda_{n, m-1}}\left(\llbracket l_{1}-1,1 \rrbracket \otimes \cdots \otimes \llbracket l_{m-2}-1,1 \rrbracket \otimes \llbracket l_{m-1} \rrbracket\right)^{S_{n}} .
\end{gathered}
$$

Remark 5.7 As already noticed, $\mathscr{S}_{n, m-2}^{\epsilon}\left(U_{m-1}\right) \varsubsetneqq \mathscr{S}_{n, m-2}^{\epsilon}\left(U_{m}\right)$, so the former set has a complement $\hat{\mathscr{S}}_{n, m-2}^{\epsilon}\left(U_{m}\right)$. Roughly speaking, this complement causes the extra-summand

$$
\bigoplus_{l \in \Lambda_{n, m-2}^{\prime}}\left(\llbracket l_{1}-1,1 \rrbracket \otimes \cdots \otimes \llbracket l_{m-2}-1,1 \rrbracket\right)^{S_{n}}
$$

to appear in the decomposition of $\Gamma_{n}^{\epsilon}\left(U_{m}\right)$, marking a difference with respect to $\Gamma_{n}^{1}\left(U_{m}\right)$. $\square$ 
Sewing together the parts we get the decomposition of $\Gamma_{n}^{L}\left(U_{m}\right)$ :

Theorem 5.8 Let $m \geqslant 3$. Then for all $n \geqslant 2$ it holds

$$
\begin{aligned}
\Gamma_{n}^{L}\left(U_{m}\right) \cong_{S_{n}} \Gamma_{n}^{L}\left(U_{m-1}\right) \oplus \bigoplus_{l \in \Lambda_{n, m-2}^{\prime}}\left(\llbracket l_{1}-1,1 \rrbracket \otimes \cdots \otimes \llbracket l_{m-2}-1,1 \rrbracket\right)^{S_{n}} \\
\oplus \bigoplus_{l \in \Lambda_{n, m-1}^{\prime}}\left(\llbracket l_{1}-1,1 \rrbracket \otimes \cdots \otimes \llbracket l_{m-1}-1,1 \rrbracket\right)^{S_{n}} \\
\oplus \bigoplus_{l \in \Lambda_{n, m-1}}\left(\llbracket l_{1}-1,1 \rrbracket \otimes \cdots \otimes \llbracket l_{m-2}-1,1 \rrbracket \otimes \llbracket l_{m-1} \rrbracket\right)^{S_{n}} .
\end{aligned}
$$

The explicit decomposition of $\Gamma_{n}^{L}\left(U_{m}\right)$ is the following:

Corollary 5.9 For all $n \geqslant 2$ it holds

$$
\begin{aligned}
& \Gamma_{n}^{L}\left(U_{m}\right) \cong S_{n} \\
& 2 \llbracket n \rrbracket \oplus 2 \bigoplus_{k=1}^{m-2} \bigoplus_{l \in \Lambda_{n, k}^{\prime}}\left(\llbracket l_{1}-1,1 \rrbracket \otimes \cdots \otimes \llbracket l_{k}-1,1 \rrbracket\right)^{S_{n}} \\
& \oplus \bigoplus_{l \in \Lambda_{n, m-1}^{\prime}}\left(\llbracket l_{1}-1,1 \rrbracket \otimes \cdots \otimes \llbracket l_{m-1}-1,1 \rrbracket\right)^{S_{n}} \\
& \oplus \bigoplus_{k=1}^{m-1} \bigoplus_{l \in \Lambda_{n, k}}\left(\llbracket l_{1}-1,1 \rrbracket \otimes \cdots \otimes \llbracket l_{k-1}-1,1 \rrbracket \otimes \llbracket l_{k} \rrbracket\right)^{S_{n}} .
\end{aligned}
$$

\section{Matrices of Small Size}

In this section we will employ the previous results to study in some details the cases $m=2$ and $m=3$. We already obtained the structure of $\Gamma_{n}^{L}\left(U_{2}\right)$ in Proposition 5.1, so the proper codimension sequence $\gamma_{n}^{L}\left(U_{2}\right):=\operatorname{dim}_{F}\left(\Gamma_{n}^{L}\left(U_{2}\right)\right)$ follows easily:

Corollary 6.1 For all $n \in \mathbb{N}$ the proper differential codimension sequence of $U_{2}$ is $\gamma_{n}^{L}\left(U_{2}\right)=n+1$.

The knowledge of the $S_{n}$-structure of $\Gamma_{n}^{L}\left(U_{2}\right)$ provides the knowledge of the $S_{n}$-structure of $P_{n}^{L}\left(U_{2}\right)$, getting back [11], Theorem 25:

Theorem 6.2 It holds $P_{n}^{L}\left(U_{2}\right) \cong_{S_{n}} \bigoplus_{\lambda \vdash n} m_{\lambda} \lambda$, where the partitions $\lambda$ involved and the corresponding multiplicities $m_{\lambda}$ are the following:

- $\lambda=\llbracket n \rrbracket$ with multiplicity $2 n+1$;

- $\lambda=\llbracket a+b, a \rrbracket$, with $a>0$ and multiplicity $3(b+1)$;

- $\lambda=\llbracket a+b+1, a+1,1 \rrbracket$ with multiplicity $b+1$. 
Proof From the decomposition of $\Gamma_{n}^{L}\left(U_{2}\right)$ and convolution formula we get

$$
\begin{aligned}
P_{n}^{L}\left(U_{2}\right) & \cong S_{n} \bigoplus_{k=0}^{n}\left(\Gamma_{k}^{L}\left(U_{2}\right) \otimes \llbracket n-k \rrbracket\right)^{S_{n}} \\
& \cong\left(\Gamma_{0}^{L}\left(U_{2}\right) \otimes \llbracket n \rrbracket\right) \oplus \bigoplus_{k=1}^{n}\left(\Gamma_{k}^{L}\left(U_{2}\right) \otimes \llbracket n-k \rrbracket\right)^{S_{n}} \\
& \cong \llbracket n \rrbracket \oplus\left(\bigoplus_{k=2}^{n}(\llbracket k-1,1 \rrbracket \otimes \llbracket n-k \rrbracket)^{S_{n}}\right) \oplus 2 \bigoplus_{k=1}^{n}(\llbracket k \rrbracket \otimes \llbracket n-k \rrbracket)^{S_{n}} .
\end{aligned}
$$

The partitions involved in the first summation are $\llbracket a+b, a \rrbracket \vdash n$ (with $a \geqslant 1$ ) and $\llbracket a+b+1, a+1,1 \rrbracket \vdash n$. By the Young-Pieri formula, the multiplicities for both are $b+1$. The Young-Pieri formula works with the second summand, as well. The partitions involved in its decomposition are $\llbracket n \rrbracket$ and $\llbracket a+b, a \rrbracket \vdash n$ (with $a \geqslant 1$ ), with multiplicities $n$ and $b+1$ respectively. Hence the total multiplicities follow immediately.

Also the differential codimensions can be computed from the proper ones (see [11], Theorem 19):

Corollary 6.3 For all $n \in \mathbb{N}$ it holds

$$
c_{n}^{L}\left(U_{2}\right)=2^{n-1}(n+2) .
$$

Proof Since $P_{n}^{L}\left(U_{2}\right) \cong S_{n} \bigoplus_{k=0}^{n}\left(\Gamma_{k}^{L}\left(U_{2}\right) \otimes \llbracket n-k \rrbracket\right)^{S_{n}}$, passing to the dimensions we get

$$
c_{n}^{L}\left(U_{2}\right)=\sum_{k=0}^{n}\left(\begin{array}{l}
n \\
k
\end{array}\right)(k-1)=2^{n-1}(n+2) \text {. }
$$

We have so far considered a faithful derivation action of $L$ on $U T_{2}(F)$; now we are going to consider the unfaithful ones.

The trivial action has of course little to offer: it amounts to consider the ordinary algebra $U T_{2}(F)$. Formally, its differential polynomial identities are generated by the differential polynomials $x^{\epsilon}$ and $x^{\delta}$ together with the ordinary polynomial $\left[x_{1}, y_{1}\right]\left[x_{2}, y_{2}\right]$. Thus the differential cocharacters and codimensions of $U T_{2}(F)$ ) differ just formally from the ordinary ones.

The remaining unfaithful actions are therefore those having the commutator Lie-ideal $L^{\prime}=F a \triangleleft L$ as kernel. So let $U_{2}^{\epsilon}$ be the $L$-algebra $U T_{2}(F)$ under the derivation action defined by $u^{a}=0$ and $u^{b}=u^{\epsilon}=\left[u, e_{22}\right]$ for all $u \in U T_{2}(F)$. Then we get

\section{Proposition 6.4 The differential polynomial identities}

$$
\left[x_{1}, x_{2}\right]\left[x_{3}, x_{4}\right], x^{\epsilon^{2}}-x^{\epsilon}, x^{\epsilon}\left[x_{1}, x_{2}\right],\left[x_{1}, x_{2}\right] x^{\epsilon},\left[x_{1}, x_{2}\right]^{\epsilon}-\left[x_{1}, x_{2}\right], x^{\delta}
$$

generate $T_{L}\left(U_{2}^{\epsilon}\right)$. The proper cocharacter sequence is

$$
\Gamma_{0}^{L}\left(U_{2}^{\epsilon}\right)=F, \Gamma_{1}^{L}\left(U_{2}^{\epsilon}\right) \cong \llbracket 1 \rrbracket \text { and, for } n \geqslant 2, \Gamma_{n}^{L}\left(U_{2}^{\epsilon}\right)=\llbracket n-1,1 \rrbracket \oplus \llbracket n \rrbracket .
$$

The proper differential codimension sequence is

$$
\gamma_{0}^{L}\left(U_{2}^{\epsilon}\right)=1 \text { and, for } n \geqslant 1, \gamma_{n}^{L}\left(U_{2}^{\epsilon}\right)=n .
$$


As before, this describes completely the differential cocharacters and codimensions of $U_{2}^{\epsilon}$

Theorem 6.5 The $S_{n}$-structure of $P_{n}^{L}\left(U_{2}^{\epsilon}\right)$ is

$$
P_{0}^{L}\left(U_{2}^{\epsilon}\right)=F, P_{1}^{L}\left(U_{2}^{\epsilon}\right) \cong \llbracket 1 \rrbracket \text { and, for all } n \geqslant 2, P_{n}^{L}\left(U_{2}^{\epsilon}\right) \cong S_{n} \sum_{\lambda \vdash n} m_{\lambda} \lambda
$$

where

- $\lambda=\llbracket n \rrbracket$ has multiplicity $n+1$;

- $\lambda=\llbracket a+b, a \rrbracket$, with $a \geqslant 1$, has multiplicity $2(b+1)$;

- $\lambda=\llbracket a+b+1, a+1,1 \rrbracket$ has multiplicity $b+1$.

The codimension sequence of $U_{2}$ is $c_{n}^{L}\left(U_{2}^{\epsilon}\right)=n 2^{n-1}+1$, for all $n \geqslant 0$.

Proof Since $P_{n}^{L}\left(U_{n}^{\epsilon}\right) \cong S_{n} \bigoplus_{k=0}^{n}\left(\Gamma_{n}^{L}\left(U_{2}^{\epsilon}\right) \otimes \llbracket n-k \rrbracket\right)^{S_{n}}$, the description of $\Gamma_{n}^{L}$ and the Pieri-Young rule provide the decomposition as in the proof of Theorem 6.2. Then, as in the proof of Corollary 6.3, just compute

$$
c_{n}^{L}\left(U_{2}^{\epsilon}\right)=\left(\begin{array}{l}
n \\
0
\end{array}\right) \gamma_{0}^{L}\left(U_{2}^{\epsilon}\right)+\sum_{k=1}^{n}\left(\begin{array}{l}
n \\
k
\end{array}\right) \gamma_{k}^{L}\left(U_{2}^{\epsilon}\right)=1+\sum_{k=1}^{n}\left(\begin{array}{l}
n \\
k
\end{array}\right) k=1+n 2^{n-1} .
$$

Once again we get back the results of [11] (Theorem 5 and Theorem 12), but with a little formal difference. Indeed, since $L / L^{\prime} \cong F b$, one can identify $U_{2}^{\epsilon}$ with the algebra $U T_{2}(F)$ endowed with a derivation action of the (commutative) Lie subalgebra $F b$ of $L$. Denoting by $T_{D}\left(U_{2}^{\epsilon}\right)$ the ideal of the differential identities of $U T_{2}(F)$ under the $F b$-derivation action, the formal differences between $T_{D}\left(U_{2}^{\epsilon}\right)$ and $T_{L}\left(U_{2}^{\epsilon}\right)$ consist solely in the presence or not of the polynomial $x^{\delta}$ among the generators. In [11] the $F b$-action has been considered, so $x^{\delta}$ is missing.

To complete the picture in the case $m=2$, in the same spirit we may consider the action of the Lie subalgebra $F a \subseteq L$ on $U T_{2}(F)$, and investigate the corresponding algebra $U_{2}^{\delta}$. We continue to denote $T_{D}\left(U_{2}^{\delta}\right)$ the ideal of differential identities of $U T_{2}(F)$, to avoid misunderstanding. The description of $T_{D}\left(U_{2}^{\delta}\right)$ is not given in [11], but can be easily recovered and very similar to the one of $T_{D}\left(U_{2}^{\epsilon}\right)$.

Proposition 6.6 The differential polynomial identities

$$
\left[x_{1}, x_{2}\right]\left[x_{3}, x_{4}\right], x^{\delta^{2}}, x^{\delta}\left[x_{1}, x_{2}\right],\left[x_{1}, x_{2}\right] x^{\delta},\left[x_{1}, x_{2}\right]^{\delta}
$$

generate $T_{D}\left(U_{2}^{\delta}\right)$. The proper cocharacter sequence is

$$
\Gamma_{0}^{D}\left(U_{2}^{\delta}\right)=F, \Gamma_{1}^{D}\left(U_{2}^{\delta}\right) \cong \llbracket 1 \rrbracket \text { and, for } n \geqslant 2, \Gamma_{n}^{D}\left(U_{2}^{\delta}\right)=\llbracket n-1,1 \rrbracket \oplus \llbracket n \rrbracket .
$$

The proper differential codimension sequence is

$$
\gamma_{0}^{D}\left(U_{2}^{\delta}\right)=1 \text { and, for } n \geqslant 1, \gamma_{n}^{D}\left(U_{2}^{\delta}\right)=n .
$$

Then, with exactly the same reasoning as for $\epsilon$, we get 
Theorem 6.7 The $S_{n}$-structure of $P_{n}^{D}\left(U_{2}^{\delta}\right)$ is

$$
P_{0}^{D}\left(U_{2}^{\delta}\right)=F, P_{1}^{D}\left(U_{2}^{\delta}\right) \cong \llbracket 1 \rrbracket \text { and, for all } n \geqslant 2, P_{n}^{D}\left(U_{2}^{\delta}\right) \cong S_{n} \sum_{\lambda \vdash n} m_{\lambda} \lambda
$$

where

- $\lambda=\llbracket n \rrbracket$ has multiplicity $n+1$;

- $\lambda=\llbracket a+b, a \rrbracket$, with $a \geqslant 1$, has multiplicity $2(b+1)$;

- $\lambda=\llbracket a+b+1, a+1,1 \rrbracket$ has multiplicity $b+1$.

The codimension sequence of $U_{2}$ is $c_{n}^{D}\left(U_{2}^{\delta}\right)=n 2^{n-1}+1$, for all $n \geqslant 0$.

It is somehow confusing the similarity between $T_{D}\left(U_{2}^{\epsilon}\right)$ and $T_{D}\left(U_{2}^{\delta}\right)$. They seem perfectly equivalent, from the point of view of their effects on the differential polynomial identities. Actually, already for $m=3$, they manifest concretely their neat differences. Let us start in describing the $S_{n}$-structure of the whole $\Gamma_{n}^{L}\left(U_{3}\right)$ :

Theorem 6.8 The $S_{n}$-decomposition of $\Gamma_{n}^{L}\left(U_{3}\right)$ is the following:

- $\Gamma_{0}^{L}\left(U_{3}\right)=F$;

- $\Gamma_{1}^{L}\left(U_{3}\right) \cong 2 \llbracket 1 \rrbracket$;

- $\quad \Gamma_{2}^{L}\left(U_{3}\right) \cong 2 \llbracket 1,1 \rrbracket \oplus 2 \llbracket 2 \rrbracket$;

- $\quad \Gamma_{3}^{L}\left(U_{3}\right) \cong 2 \llbracket 3 \rrbracket \oplus 3 \llbracket 2,1 \rrbracket \oplus \llbracket 1,1,1 \rrbracket$

and, for $n \geqslant 4$,

$$
\begin{aligned}
\Gamma_{n}^{L}\left(U_{3}\right) & \cong 2 \llbracket n \rrbracket \oplus(n+1) \llbracket n-1,1 \rrbracket \oplus \bigoplus_{a \geqslant 2} 2(b+1) \llbracket a+b, a \rrbracket \oplus(2 n-5) \llbracket n-2,1,1 \rrbracket \\
& \oplus \bigoplus_{a \geqslant 1} 3(b+1) \llbracket 1+a+b, 1+a, 1 \rrbracket \oplus \underset{a \geqslant 0}{\bigoplus}(b+1) \llbracket 2+a+b, 2+a, 2 \rrbracket \\
& \oplus \bigoplus_{a \geqslant 0}(b+1) \llbracket 1+a+b, 1+a, 1,1 \rrbracket .
\end{aligned}
$$

Proof Actually, a proof is not needed, since the general result given in Theorem 5.8. Nevertheless, a guideline for the concrete decomposition of $\Gamma_{n}^{L}\left(U_{3}\right)$ may be of help. The cases $n \leqslant 3$ are straightforward, so assume $n \geqslant 4$. We know the decomposition

$$
\Gamma_{n}^{L}\left(U_{2}\right)=2 \cdot \llbracket n \rrbracket \oplus \llbracket n-1,1 \rrbracket .
$$

The set $\Lambda_{n, 1}^{\prime}$ has cardinality 1 , since just $(n)$ is a weak 1-composition of $n$. So the summand indexed by $\Lambda_{n, 1}^{\prime}$ in Theorem 5.8 is simply $\llbracket n-1,1 \rrbracket$.

Then the set $\Lambda_{n, 2}^{\prime}$ consists of all pairs $\left(l_{1}, l_{2}\right)$ such that $l_{1}, l_{2} \geqslant 2$, so it is empty if $n \leqslant 3$. If $n \geqslant 4$ there are exactly $n-3$ such compositions, and each of them gives rise to the module $\left(\llbracket l_{1}-1,1 \rrbracket \otimes \llbracket l_{2}-1,1 \rrbracket\right)^{S_{n}}$. Let $U\left(\Lambda_{n, 2}^{\prime}\right)$ be the sum of these $S_{n}$-modules. Then, 
the Littlewood-Richardson rule provides the decomposition

$$
\begin{aligned}
U\left(\Lambda_{n, 2}^{\prime}\right) & \cong \bigoplus_{a \geqslant 2}(b+1) \cdot \llbracket a+b, a \rrbracket \\
& \oplus(n-3) \cdot \llbracket n-2,1,1 \rrbracket \oplus \bigoplus_{a \geqslant 1}^{2} 2(b+1) \cdot \llbracket 1+a+b, 1+a, 1 \rrbracket \\
& \oplus \bigoplus_{a \geqslant 0}(b+1) \cdot \llbracket 2+a+b, 2+a, 2 \rrbracket \oplus \bigoplus_{a \geqslant 0}(b+1) \cdot \llbracket 1+a+b, 1+a, 1,1 \rrbracket .
\end{aligned}
$$

Finally, the set $\Lambda_{n, 2}$ consists of all pairs $\left(l_{1}, l_{2}\right)$ such that $l_{1}+l_{2}=n, l_{1} \geqslant 2$ and $l_{2} \geqslant 1$. It is empty if $n<3$, but if $n \geqslant 3$ it consists of $n-2$ pairs, each of them giving rise to the $S_{n}$-module $\left(\llbracket l_{1}-1,1 \rrbracket \otimes \llbracket l_{2} \rrbracket\right)^{S_{n}}$. If $U\left(\Lambda_{n, 2}\right)$ denotes the sum of all such $S_{n}$-modules, the Pieri-Young formula provides the decomposition

$$
\begin{aligned}
U\left(\Lambda_{n, 2}\right) & \cong(n-1) \cdot \llbracket n-1,1 \rrbracket \oplus \bigoplus_{a \geqslant 2}(b+1) \cdot \llbracket a+b, a \rrbracket \\
& \oplus(n-2) \cdot \llbracket n-2,1,1 \rrbracket \oplus \bigoplus_{a \geqslant 1}(b+1) \cdot \llbracket 1+a+b, 1+a, 1 \rrbracket .
\end{aligned}
$$

Now the $S_{n}$-structure of $\Gamma_{n}^{L}\left(U_{3}\right)$ follows simply summing the multeplicities of the involved irreducible $S_{n}$-modules.

Remark 6.9 It may be useful to distinguish among the irreducible components of $\Gamma_{n}^{1}\left(U_{3}\right)$, $\Gamma_{n}^{\delta}\left(U_{3}\right)$ and $\Gamma_{n}^{\epsilon}\left(U_{3}\right)$, at least when $n \geqslant 4$. We have

$$
\begin{aligned}
\Gamma_{n}^{1}\left(U_{3}\right) & \cong \llbracket n-1,1 \rrbracket \oplus \bigoplus_{a \geqslant 2}(b+1) \llbracket a+b, a \rrbracket \\
& \oplus(n-3) \llbracket n-2,1,1 \rrbracket \oplus \bigoplus_{a \geqslant 1} 2(b+1) \llbracket 1+a+b, 1+a, 1 \rrbracket \\
& \oplus \bigoplus_{a \geqslant 0}(b+1) \llbracket 2+a+b, 2+a, 2 \rrbracket \oplus \bigoplus_{a \geqslant 0}(b+1) \llbracket 1+a+b, 1+a, 1,1 \rrbracket \\
\Gamma_{n}^{\delta}\left(U_{3}\right) & \cong \llbracket n \rrbracket \\
\Gamma_{n}^{\epsilon}\left(U_{3}\right) & \cong \llbracket n \rrbracket \oplus n \llbracket n-1,1 \rrbracket \oplus \bigoplus_{a \geqslant 2}(b+1) \llbracket a+b, a \rrbracket \\
& \oplus(n-2) \llbracket n-2,1,1 \rrbracket \oplus \bigoplus_{a \geqslant 1}(b+1) \llbracket 1+a+b, 1+a, 1 \rrbracket .
\end{aligned}
$$

This makes evident the different impact of $\epsilon$ and $\delta$ on differential polynomial identities of $U_{m}$ in the general cases, that is for $m>2$ (see the proof of Proposition 5.1). Taking $\Gamma_{n}^{1}\left(U_{3}\right) \cong{ }_{n} \Gamma_{n}\left(U T_{3}(F)\right)$ as the pillar of the comparison, the $S_{n}$-structures of $\Gamma_{n}^{D}\left(U_{3}^{\epsilon}\right)$ and $\Gamma_{n}^{D}\left(U_{3}^{\delta}\right)$ are greatly different:

$$
\begin{aligned}
& \Gamma_{n}^{D}\left(U_{3}^{\delta}\right) \cong S_{n} \Gamma_{n}\left(U T_{3}(F)\right) \oplus \llbracket n \rrbracket \\
& \Gamma_{n}^{D}\left(U_{3}^{\epsilon}\right) \cong S_{n} \quad \Gamma_{n}\left(U T_{3}(F)\right) \oplus \llbracket n \rrbracket \oplus n \llbracket n-1,1 \rrbracket \oplus \bigoplus_{a \geqslant 2}(b+1) \llbracket a+b, a \rrbracket \\
& \\
& \oplus \quad(n-2) \llbracket n-2,1,1 \rrbracket \oplus \bigoplus_{a \geqslant 1}(b+1) \llbracket 1+a+b, 1+a, 1 \rrbracket
\end{aligned}
$$


To put it differently, the $S_{n}$-module $\Gamma_{n}^{D}\left(U_{3}^{\epsilon}\right)$ is almost the whole $\Gamma_{n}^{L}\left(U_{3}\right)$, just an occurrence of the single one-dimensional module $\llbracket n \rrbracket$ is missing. On the other extreme, $\Gamma_{n}^{D}\left(U_{3}^{\delta}\right)$ is little more than $\Gamma_{n}\left(U T_{3}(F)\right)$ : just an extra occurence of the one-dimensional module $\llbracket n \rrbracket$ is added.

Corollary 6.10 The proper differential codimension sequence of $U_{3}$ is

$$
\begin{gathered}
\gamma_{0}^{L}\left(U_{3}\right)=1, \gamma_{1}^{L}\left(U_{3}\right)=2, \gamma_{2}^{L}\left(U_{3}\right)=4, \gamma_{3}^{L}\left(U_{3}\right)=9, \\
\text { and, for } n \geqslant 4, \quad \gamma_{n}^{L}\left(U_{3}\right)=n(n-3) 2^{n-2}+3 n .
\end{gathered}
$$

Proof It is awkward to compute $\gamma_{n}^{L}\left(U_{3}\right)$ from the $S_{n}$-structure of $\Gamma_{n}^{L}\left(U_{3}\right)$ presented above when $n \geqslant 4$. A more convenient way is to use the decomposition into induced tensor products provided by Theorem 5.8. We already know that $\gamma_{n}^{L}\left(U_{2}\right)=n+1$; moreover since $\Lambda_{n, 1}^{\prime}=\{(n)\}$ the composite module relative to $\Lambda_{n, 1}^{\prime}$ consists of the single irreducible module $\llbracket n-1,1 \rrbracket$ of dimension $n-1$.

Next, each summand $\left(\llbracket l_{1}-1,1 \rrbracket \otimes \llbracket l_{2}-1,1 \rrbracket\right)^{S_{n}}$ associated to $\left(l_{1}, l_{2}\right) \in \Lambda_{n, 2}^{\prime}$ has dimension $\left(l_{1}-1\right)\left(l_{2}-1\right)\left(\begin{array}{l}n \\ l_{1}\end{array}\right)$, so their direct sum has dimension

$$
\sum_{l=2}^{n-2}\left(\begin{array}{l}
n \\
l
\end{array}\right)(l-1)(n-l-1)=(n-1)(n-4) 2^{n-2}+2(n-1) .
$$

Finally, considering the last composite module corresponding to the set $\Lambda_{n, 2}$, each of its summands $\left(\llbracket l_{1}-1,1 \rrbracket \otimes \llbracket l_{2} \rrbracket\right)^{S_{n}}$ has dimension $\left(l_{1}-1\right)\left(\begin{array}{l}n \\ l_{1}\end{array}\right)$, hence the direct sum corresponding to $\Lambda_{n, 2}$ has dimension

$$
\sum_{l=2}^{n-1}\left(\begin{array}{l}
n \\
l
\end{array}\right)(l-1)=(n-2)\left(2^{n-1}-1\right) .
$$

Then $\gamma_{n}^{L}\left(U_{3}\right)=\operatorname{dim} \Gamma_{n}^{L}\left(U_{3}\right)$ is simply the sum of these dimensions.

We must remark that just $\gamma_{0}^{L}\left(U_{3}\right)$ does not match the general formula.

In principle, the explicit decomposition of $P_{n}^{L}\left(U_{3}\right)$ follows from the Pieri-Young formula and the decomposition of $\Gamma_{n}^{L}\left(U_{3}\right)$, that is

$$
P_{n}^{L}\left(U_{3}\right) \cong \bigoplus_{k=0}^{n}\left(\Gamma_{k}^{L}\left(U_{3}\right) \otimes \llbracket n-k \rrbracket\right)^{S_{n}} .
$$

By the way, its explicit description is little interesting while quite complex. It is more useful to measure the complexity of $P_{n}^{L}\left(U_{3}\right)$ through its codimension sequence:

Corollary 6.11 The differential codimension sequence of $U_{3}$ is the following:

$$
c_{0}^{L}\left(U_{3}\right)=1 \quad \text { and, for } n \geqslant 1, c_{n}^{L}\left(U_{3}\right)=n(n-4) 3^{n-2}+3 n \cdot 2^{n-1}+1
$$

Proof It follows from $c_{n}^{L}\left(U_{3}\right)=\sum_{k=0}^{n}\left(\begin{array}{l}n \\ k\end{array}\right) \gamma_{k}^{L}\left(U_{3}\right)$ by straightforward computations, and just the first term $\gamma_{0}^{L}\left(U_{3}\right)$ needs to be directly set to 1 , while $\gamma_{k}$ is exactly the one provided by the general formula in Corollary 6.10 .

For the sake of completeness, we record 
Corollary 6.12 The codimension sequences $c_{n}^{D}\left(U_{3}^{\delta}\right)$ and $c_{n}^{D}\left(U_{3}^{\epsilon}\right)$ are the following:

$$
\begin{array}{cl}
\text { for } n=0: & c_{0}^{D}\left(U_{3}^{\delta}\right)=c_{0}^{D}\left(U_{3}^{\epsilon}\right)=c_{0}^{L}\left(U_{3}\right)=1 \\
\text { for } n \geqslant 1: & c_{n}^{D}\left(U_{3}^{\delta}\right)=3^{n-2}\left(n^{2}-7 n+9\right)+(n-1) 2^{n}+\frac{1}{6}\left(2 n^{3}-3 n^{2}+n+6\right) \\
& c_{n}^{D}\left(U_{3}^{\epsilon}\right)=n(n-4) 3^{n-2}+(3 n-2) \cdot 2^{n-1}+2
\end{array}
$$

Proof Concrete calculations are needed, but they can be minimized computing at first the basic (ordinary) codimension $c_{n}:=c_{n}\left(U T_{3}(F)\right)$. Setting $\gamma_{n}:=\gamma_{n}\left(U T_{3}(F)\right)$, one easily has

$$
\gamma_{0}=1, \quad \gamma_{1}=0, \gamma_{2}=1, \quad \gamma_{3}=2
$$

and then, for $n \geqslant 4$, it holds

$$
\gamma_{n}=\sum_{k=2}^{n-2}\left(\begin{array}{l}
n \\
k
\end{array}\right)(k-1)(n-k-1)=(n-1)(n-4) 2^{n-2}+2(n-1) .
$$

This general formula does not match the lower numbers $\gamma_{n}$ for $n=0,1,2,3$. More precisely, it evaluates to zero for $n=1,2,3$. Therefore one can compute directly

$$
c_{0}=1, \quad c_{1}=1, \quad c_{2}=2, \quad c_{3}=6
$$

and then, for $n \geqslant 4$, one has

$$
\begin{aligned}
c_{n} & =\left(\begin{array}{l}
n \\
0
\end{array}\right) \gamma_{0}+\left(\begin{array}{l}
n \\
1
\end{array}\right) \gamma_{1}+\left(\begin{array}{l}
n \\
2
\end{array}\right) \gamma_{2}+\left(\begin{array}{l}
n \\
3
\end{array}\right) \gamma_{3}+\sum_{k=4}^{n}\left(\begin{array}{l}
n \\
k
\end{array}\right) \gamma_{k} \\
& =1+\frac{n(n-1)}{2}+\frac{n(n-1)(n-2)}{3}+\sum_{k=4}^{n}\left(\begin{array}{l}
n \\
k
\end{array}\right)\left(k^{2} 2^{k-2}-5 k 2^{k-2}+2^{k}+2 k-2\right) \\
& =\frac{3^{n-2}}{2}\left(2 n^{2}-14 n+18\right)+(n-2) 2^{n}+\frac{1}{6}\left(2 n^{3}-3 n^{2}+n+12\right) .
\end{aligned}
$$

Now just note that

$$
\gamma_{0}^{D}\left(U_{2}^{\delta}\right)=1=\gamma_{0} \text { and, for all } n \geqslant 1, \gamma_{n}^{D}\left(U_{3}^{\delta}\right)=\gamma_{n}+1,
$$

because just the single one-dimensional module $\llbracket k \rrbracket$ joins the decomposition of $\Gamma_{n}\left(U T_{3}(F)\right)$ to get the decomposition of $\Gamma_{n}^{D}\left(U_{3}^{\delta}\right)$, so

$$
c_{n}^{D}\left(U_{3}^{\delta}\right)=c_{n}+\sum_{k=1}^{n}\left(\begin{array}{l}
n \\
k
\end{array}\right)=c_{n}+2^{n}-1
$$

is the $n$-th differential codimension of $U_{3}^{\delta}$. Similar arguments work for $c_{n}^{D}\left(U_{3}^{\epsilon}\right)$ : now the starting point is $c_{n}^{L}\left(U_{3}\right)$, and just notice that $c_{0}^{L}\left(U_{3}\right)=c_{0}^{D}\left(U_{3}^{\epsilon}\right)$ while for $n \geqslant 1$ from $\gamma_{n}^{L}\left(U_{3}\right)=\gamma_{n}^{D}\left(U_{3}^{\epsilon}\right)+1$ (since just the one-dimensional module $\llbracket n \rrbracket$ is missing to get $\Gamma_{n}^{L}\left(U_{3}\right)$ from the decomposition of $\left.\Gamma_{n}^{D}\left(U_{3}^{\epsilon}\right)\right)$ it follows

$$
c_{n}^{D}\left(U_{3}^{\epsilon}\right)=c_{n}^{L}\left(U_{3}\right)-\sum_{k=1}^{n}\left(\begin{array}{l}
n \\
k
\end{array}\right)=c_{n}^{L}\left(U_{3}\right)-\left(2^{n}-1\right) .
$$

Funding Open Access funding provided by Università degli Studi di Bari "Aldo Moro" 
Open Access This article is licensed under a Creative Commons Attribution 4.0 International License, which permits use, sharing, adaptation, distribution and reproduction in any medium or format, as long as you give appropriate credit to the original author(s) and the source, provide a link to the Creative Commons licence, and indicate if changes were made. The images or other third party material in this article are included in the article's Creative Commons licence, unless indicated otherwise in a credit line to the material. If material is not included in the article's Creative Commons licence and your intended use is not permitted by statutory regulation or exceeds the permitted use, you will need to obtain permission directly from the copyright holder. To view a copy of this licence, visit http://creativecommonshorg/licenses/by/4.0/.

\section{References}

1. Bahturin, Yu.A.: Identical Relations in Lie Algebras. VNU Science Press, Utrecht (1987)

2. Bahturin, Y.A., Yasumura, F.: Distinguishing simple algebras by means of polynomial identities. São Paulo J. Math. 13, 39-72 (2019)

3. Beidar, K.I., Martindale, I., W. S., Mikhalev, A.V.: Rings with generalized identities. Marcel Dekker, Inc, (1996)

4. Berele, A.: Cocharacter sequence for algebras with Hopf algebra actions. J. Algebra 185, 869-885 (1996)

5. Coelho, S.P., Polcino Milies, C.: Derivations of upper triangular matrix rings. Linear Algebra Appl. 187, 263-267 (1993)

6. Di Vincenzo, O.M., Nardozza, V.: On the $*$-Polynomial Identities of a Class of $*$-minimal algebras. Comm. Algebra 38, 3078-3093 (2010)

7. Drensky, V.: Free Algebras and PI-Algebras. Graduate course in Algebra. Springer, Singapore (2000)

8. Drensky, V., Giambruno, A.: Cocharacters, codimensions and Hilbert series of the polynomial identities for $2 \times 2$ matrices with involution. Canad. J. Math. 46, 718-733 (1994)

9. Drensky, V., Kasparian, A.: Polynomial identities of eighth degree for $3 \times 3$ matrices, Annuaire de l'Univ. de Sofia. Fac. de Math. et Meca., Livre 1, Math. 77, 175-195 (1983)

10. Galitski, L.Yu., Timashev, D.A.: On classification of metabelian Lie algebras. J. Lie Theory 9, 125-156 (1999)

11. Giambruno, A., Rizzo, C.: Differential identities, $2 \times 2$ upper triangular matrices and varieties of almost polynomial growth, J. Pure Appl. Algebra. https://doi.org/10.1016/j.jpaa.2018.07.004 (2018)

12. Jacobson, N.: Lie algebras. Interscience Publishers (1962)

13. Karchenko, V.K.: Differential identities of prime rings. Algebra and Logic 17(2), 58-80 (1979)

Publisher's Note Springer Nature remains neutral with regard to jurisdictional claims in published maps and institutional affiliations. 